Sonderabdruck aus

SCHWEIZERISCHE ZEITSCHRIFT FUR HYDROLOGIE BIRKHAUUSER VERLAG BASEL

Vol. XX $1958 \quad$ Fasc. 2

\title{
Untersuchungen über die bakterizide Wirkung von Silber in Wasser
}

Von K. WUHRMANN und F. $Z \varrho$ BRIST

Mitteilung Nr. I42 der Eidg. Anstalt für Wasserversorgung, Abwasserreinigung und Gewässerschutz an der E'TH, Zürich. Direktor: Prof. Dr. O. JAAG 


\title{
Untersuchungen über die bakterizide Wirkung von Silber in Wasser
}

\author{
Von K. WUHRMANN und F. ZOBRIST \\ Mitteilung Nr. I42 der Eidg. Anstalt für Wasserversorgung, Abwasserreinigung \\ und Gewässerschutz an der ETH, Zürich. Direktor: Prof. Dr. O. JAAG
}

Manuskript eingegangen am 22. Mai 1958

\section{Einleitung}

Für die Beurteilung eines Desinfektionsmittels in der Wasseraufbereitungstechnik ist die genaue Kenntnis der Beziehung zwischen der Konzentration des Desinfektionsmittels und der Abtötungsgeschwindigkeit der in Frage kommenden Organismen unter den verschiedenen, in der Praxis massgebenden äusseren Einflüssen, wie Wasserchemismus, Temperatur, $\mathrm{pH}$, Sauerstoffgehalt usw., als Grundlage zu betrachten. Während bei anderen praktisch verwendeten Desinfektionsmitteln, wie Chlor, Chlordioxyd, Ozon, diese Beziehung wenigstens bei einigen Bedingungen quantitativ bekannt ist, beschränken sich unsere Informationen beim Silber auf eine grosse Zahl qualitativer Angaben, die sich häufig widersprechen oder offensichtlich unrichtig sind, wenn man ihre experimentellen Grundlagen prüft. Wir verweisen in diesem Zusammenhang auf neuere Literaturzusammenstellungen bei Goetz et al. [5] $]^{1}$ ), ZLMMERMANN [19], Romans [I4] und OBERZILL [Io].

Im Hinblick auf die praktischen Bedürfnisse haben wir den Untersuchungen, die in den Jahren 1954 bis 1956 vorgenommen wurden, zunächst folgende Aufgaben gestellt:

I. Es sind die methodischen Grundlagen zu schaffen, um reproduzierbare und hinsichtlich aller äusseren Faktoren genau kontrollierte Abtötungsversuche mit Silber durchführen zu können.

2. Es ist der Einfluss folgender Faktoren auf die Wirksamkeit von Silber bei der Desinfektion von Wasser quantitatio festzustellen:

\footnotetext{
2) Die Ziffern in eckigen Klammern verweisen auf das Literaturverzeichnis, Seite 253.
} 
Herkunft und Konzentration des Silbers;

Einfluss wichtiger Kationen und Anionen in Wasser $\left(\mathrm{pH}, \mathrm{Ca}^{+2}, \mathrm{Cl}^{-}\right.$, $\mathrm{PO}_{4}^{+3}$ );

Temperatur;

Bakterienzahl, -alter, -art;

Sauerstoffgehalt.

Aus allen älteren Untersuchungen und den meisten praktischen Erfahrungen geht hervor, dass Silber (auch in hohen Konzentrationen) im Vergleich mit anderen Desinfektionsmitteln relativ langsam auf Bakterien einwirkt. Es können vorläufig nur Vermutungen über die Ursache der geringen Geschwindigkeiten sichtbarer Silberwirkungen auf Organismen geäussert werden. Jedenfalls wagen wir es nicht, aus unseren bisherigen Beobachtungen Schlüsse auf die Art und die Beeinflussbarkeit der einzelnen Teilvorgänge zu ziehen, die ablaufen müssen, um den Tod einer in Ag-Lösung suspendierten Zelle zu verursachen. Es herrscht heute die Meinung vor, dass die bakteriziden Partikel in Silberlösungen allein durch die Silberionen $\mathrm{Ag}^{+}$repräsentiert werden (GoETZ et al. [5], GAUZE [4]). Zahlreiche unserer Feststellungen lassen uns an dieser Auffassung zweifeln, doch müssten zur genaueren Abklärung dieser äusserst komplizierten Verhältnisse noch eine grosse Reihe speziell angelegter Versuche vorgenommen werden. Wir gehen vorläufig davon aus, dass die tödliche Wirkung von Silber auf eine Zelle schlussendlich in einer Blockierung enzymatischer Reaktionen besteht, das heisst nur von Silberteilchen erreicht werden kann, welche noch zu Reaktionen mit Zellbestandteilen befähigt sind. Ob dazu nur Ag-Ionen in der Lage sind, ist eher unwahrscheinlich. Unsere Messungen stellen zunächst lediglich Korrelationen zwischen der analytisch messbaren Gesamtkonzentration von Silber in wässrigen Lösungen und dem Abtötungseffekt dar, ohne dass wir über Ladungszustand, Partikelgrösse oder die Zusammensetzung eventuell vorhandener komplexer $\mathrm{Ag}$-Verbindungen Genaueres wissen.

\section{Methodische Grundlagen}

\section{I. Silberbestimmung}

Bei vielen bisher veröffentlichten Desinfektionsversuchen von Wasser mit Silber ist die effektiv wirksam gewesene Silberkonzentration nicht genau bekannt, da sie analytisch überhaupt nicht oder dann mit unzulänglichen Methoden bestimmt wurde. Im weitern erfolgten die Experimente 
häufig unter Bedingungen, bei welchen die zugesetzte aktive Silbermenge entweder durch Umsetzung mit Substanzen aus den Bakterien-Nährböden oder durch Adsorption an die Wände der Versuchsgefässe im Laufe der Versuchszeit in unbekanntem Umfange gebunden wurde.

Alle geprüften Sorten von Laboratoriumsgläsern, mit Ausnahme von Pyrex und reinem Quarz, sind wegen ihrer starken Silberadsorption unbrauchbar für genauere Experimente mit Silberlösungen. Gewisse Sorten von Polyäthylen, die für Laborgeräte verarbeitet werden, zeigen ebenfalls eine praktisch vernachlässigbare Silberadsorption.

Nach eingehenden vergleichenden Untersuchungen verschiedener Silberbestimmungsmethoden in natürlichen Wässern erwiesen sich folgende Verfahren als zweckmässig:

a) Durch eine Kolonne (Durchmesser maximal Io $\mathrm{mm}$ ), welche mit $\mathrm{I}, 5-2 \mathrm{ml}$ Dowex-5o beladen ist, wird so viel silberhaltiges Wasser durchperkoliert, dass der Kationenaustauscher mit mindestens $5 \mu \mathrm{g}$ Silber beschickt ist. Fliessgeschwindigkeit etwa IO-I5 Tropfen pro Io s. Der Kationenaustauscher wird nun quantitativ in einen Scheidetrichter gestossen, mit destilliertem Wasser nachgespült, und dann das Wasser durch leichtes Öffnen des Hahns abfliessen gelassen. Sodann werden Io $\mathrm{ml}$ destilliertes Wasser und $0,5 \mathrm{ml} 4 \mathrm{nHNO}_{3}$ zugefügt und gemäss den Angaben von FISCHER und LEOPOLDI [3] mit Dithizon titriert.

Bei den letzten kleinen Reagenszusätzen tritt bisweilen eine schlechte Schichtentrennung ein. Die Dithizon-Lösung liegt als feine Suspension zwischen den Körnern des Harzes und kann durch langsames Drehen des Scheidetrichters im konischen Teil angesammelt werden. Fliessen die Tröpfchen dennoch nicht zusammen, so kann mit der Spitze eines ausgezogenen Glasstabes, die vorher in Octylalkohol getaucht wurde, nachgeholfen werden. Sämtliche zur Analyse verwendeten Glasutensilien bestehen aus Pyrexglas. Die Methode gestattet den quantitativen Nachweis von Silber im Konzentrationsbereich von I bis etwa $\mathrm{r} 5 \mathrm{O} \mu \mathrm{g} / \mathrm{l}$. Genauigkeit bei weniger als Io $\mu \mathrm{g} / 1$ rund $\pm 4 \%$. Bei über Io $\mu \mathrm{g} / 1 \pm 2 \%$.

Wir möchten an dieser Stelle auf eine seither in unserm Laboratorium von Herrn Dr. v. BEust ${ }^{2}$ ) ausgearbeitete Variante der Silberbestimmungsmethode hinweisen, welche das Titrieren durch tropfenweises Ausschütteln des Wassers mit Dithizon vermeidet und sich ausgezeichnet bewährt hat:

b) Dithizon in $\mathrm{CCl}_{4}$ besitzt bei $625 \mathrm{~m} \mu$ ein ausgeprägtes Absorptionsmaximum, während der Ag-Dithizonkomplex völlig durchlässig ist (Ab-

*) Wir verdanken Herrn Dr. v. Beust die Mitarbeit an diesem Problem bestens. 
sorptionsmaximum bei $470 \mathrm{~m} \mu$ ). Aus der Absorptionsabnahme einer Dithizonlösung bei $625 \mathrm{~m} \mu$ bei Anwesenheit von Silber lässt sich deshalb die an der Komplexbildung beteiligte Silbermenge quantitativ ermitteln. Verfabren: Je nach dem Silbergehalt des Wassers werden $50-500 \mathrm{ml}$ in einen Schütteltrichter passender Grösse gegossen und mit Salpetersäure versetzt ( $4 \mathrm{n}, 0,3 \mathrm{ml}$ pro Io $\mathrm{ml}$ Wasser). Dann lässt man genau $5 \mathrm{ml}$ einer Dithizonlösung in $\mathrm{CCl}_{4}$ aus einer Bürette zufliessen und schüttelt eine Minute lang intensiv. Dann lässt man die beiden Phasen trennen und überführt die Dithizonlösung in eine ro-mm-Messküvette (Beckmann-DU-Spektrophotometer). In die Referenzküvette gibt man von der ursprünglichen Dithizonlösung. Die Proben werden mit einigen Tropfen Wasser überschichtet, um das Verdunsten des $\mathrm{CCl}_{4}$ zu verhindern. Die Absorptionsdifferenz der beiden Lösungen wird bei $625 \mathrm{~m} \mu$ bestimmt.

Die Reinigung des Dithizons und Herstellung der Lösung erfolgt nach Fischer und LeOPoldi [3]. Die Dithizonkonzentration wird so gewählt, dass die Extinktion der Lösung gegen reinen $\mathrm{CCl}_{4}$ ungefähr $\mathrm{I}$, o beträgt (entspricht etwa 3,3 $\mu \mathrm{g} \mathrm{Ag} / \mathrm{ml}$ DTZ). Messbereich: I-300 $\mu \mathrm{g}$ oder mehr $\mathrm{Ag} / \mathrm{l}$ Wasser. Störungen können auftreten, wenn gleichzeitig Kupfer anwesend ist. Sie werden am sichersten vermieden, wenn man anstelle des Dithizons den Cu-Dithizonkomplex als Silberreagens verwendet. Im sauren Milieu verdrängt nämlich das Silber das Kupfer, und man kann dann die Extinktion des Ag-Dithizonkomplexes direkt bei 470 m $\mu$ messen. Zink stört die Silberbestimmung nicht, weil das Zn-Dithizon im sauren Bereich nicht gebildet wird.

\subsection{Bakteriologische Methoden}

Die nachfolgend beschriebenen Experimente erfolgten fast ausschliesslich nach dem folgenden Schema:

a) Test-Bakterien: Vorkultur in flüssigen Nährböden (bei E. coli: $48 \mathrm{~h}$ in Milchzuckerbouillon). Mehrmaliges Waschen der Bakterien in steriler Pufferlösung (mittels Zentrifugieren) und Herstellen einer Suspension gewünschter Keimdichte in Pufferlösung durch Verdünnung.

b) Einpipettieren der notwendigen Menge Bakteriensuspension (meist I $\mathrm{ml}$ ) in die vorher vorbereitete Silberlösung, die bereits alle eventuell zu prüfenden Salze enthielt und auf das gewünschte $\mathrm{pH}$ und die Versuchstemperatur eingestellt war.

c) Bei jeder geprüften Kontaktzeit wurden 2 Proben zu je $\mathrm{I} \mathrm{cm}^{3}$ aus der Silberlösung erhoben und sofort in üblicher Weise in Petrischalen zusammen 
mit einer Agarnährlösung ausgesät. Nährboden für Versuche mit E. coli: Glukose-Pepton-Agar. Bebrütung bei $37^{\circ}$, Auszählung nach 24 bis $48 \mathrm{~h}$.

d) Als massgebende Anfangskeimzahl galt das Zählungsresultat von den Platten der Doppelproben, die unmittelbar nach Vermischen der Impfsuspension mit der Silberlösung erhoben wurden.

e) Sämtliche in Kontakt mit wässrigen Silberlösungen gelangenden Geräte bestanden aus Pyrexglas.

f) Bei den meisten Experimenten erfolgte nach der letzten Probenahme eine Kontrollbestimmung des Silbergehaltes der Desinfektionslösung. Stimmte die gefundene Ag-Konzentration am Schluss des Versuches nicht mit der Anfangskonzentration überein, so wurde der Versuch verworfen.

g) Die Zahl der auf den Agarplatten auswachsenden Kolonien wurde der Anzahl noch lebender Keime im Moment der Probenahme gleichgesetzt. Dieses Verfahren kann nur ein richtiges Bild über die Abtötung der Bakterien ergeben, wenn eineSilber-Nachwirkung in den Nährböden ausgeschlossen ist.

Nun ist wohl bekannt, dass Sulfide und organische Substanzen mit sulfidischen Gruppen (zum Beispiel Cystin, Thioglykolsäure, Glutathion usw.) mit Silber sofort reagieren und es in sulfidischer Bindung fixieren. Mit äquivalenten Mengen von Sulfidbildnern, die auch in den üblichen Nährlösungen vorhanden sind, kann eine Silberlösung momentan «entgiftet» werden. Das Beispiel in Tabelle I zeigt, dass das Absterben von E. coli in einer Ag-Lösung nach Zusatz von Bakteriennährlösung der üblichen Konzentration tatsächlich sofort unterbrochen wird. Der Effekt hängt selbst-

Tabelle 1

Bakterizide Wirkung pon Ag+ auf E. coli in Milcbzucker-Bouillon

\begin{tabular}{|c|c|c|c|c|c|c|}
\hline & \multicolumn{6}{|c|}{$\%$ Überlebende nach } \\
\hline & 0 & 10 & 20 & 30 & 45 & $60 \mathrm{~min}$ \\
\hline 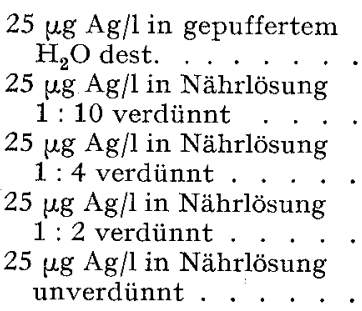 & $\begin{array}{l}100 \\
100 \\
100 \\
100 \\
100\end{array}$ & 57,7 & 22,2 & $\begin{array}{r}6,1 \\
84,0 \\
101\end{array}$ & 1,3 & $\begin{array}{r}0,1 \\
64,6 \\
104 \\
101 \\
101\end{array}$ \\
\hline
\end{tabular}


verständlich von den Mengenverhältnissen zwischen Nährlösung und AgKonzentration ab (die Verdünnung I : Io führt zum Beispiel noch nicht zu einer vollständigen Inaktivierung von $25 \mu \mathrm{g} \mathrm{Ag} / \mathrm{l}$ ). Innerhalb des bisher geprüften Bereiches von Silberkonzentrationen konnte aber mit restloser Silberbindung durch die Nährbodensubstanzen gerechnet werden, ohne dass spezielle Inaktivierungsmittel zugesetzt wurden.

Bei dieser Gelegenheit weisen wir darauf hin, dass bei Untersuchungen in der Praxis, wo zwischen Probenahme bei einer Wasserversorgung und der Verarbeitung der Proben längere Zeit verstreichen kann, die Ag-Nachwirkungen während des Probentransportes durch geeignete Massnahmen zu verhindern sind.

\subsection{Auswertung der Bakterienzählungen}

Die vergleichende Prüfung des Einflusses verschiedener Faktoren auf die Desinfektionswirkung von Silberlösungen ist in exakter Weise nur möglich, wenn die Streuungen, die allen biologischen Testen eigen sind, gebührend berücksichtigt werden. Man benötigt deshalb für Wirkungsvergleiche möglichst einfache Parameter, deren Streuungen rechnerisch erfassbar sind, so dass sie einer statistischen Bearbeitung zugänglich werden. Die Wirkung eines Desinfektionsmittels ist durch folgende stetige Beziehungen charakterisierbar:

Absterbegeschwindigkeit einer Bakterienpopulation, das heisst Funktion zwischen der Zahl überlebender Bakterien und Kontaktzeit bei bestimmter Silberkonzentration und unter bestimmten äusseren Bedingungen. Aus der Absterbegeschwindigkeit lassen sich gegebenenfalls Geschwindigkeitskonstanten für die Bakterienabtötung errechnen, die besonders geeignet sind, bei ein und demselben Desinfektionsmittel die Wirkung verschiedener äusserer Faktoren zu vergleichen.

Konzentrations-Kontaktzeit-Funktion für eine bestimmte Abtötungsrate aller Bakterien, zum Beispiel 99,9\% Abtötung, bei bestimmten Bedingungen. Diese stetige Beziehung lässt sich aus Absterbekurven ermitteln. Die Konzentrations-Kontaktzeit-Funktion liefert die für die Dimensionierung von Desinfektionsanlagen benötigten Ausgangswerte und ist ferner zum Vergleich verschiedener Desinfektionsverfahren besonders geeignet.

\subsection{Absterbeordnung pon Bakterien in Silberlösungen}

Bei der Einwirkung von Silber auf eine Bakterienpopulation vermindert sich die Zahl lebender Keime in Abhängigkeit der Kontaktzeit gemäss einer 


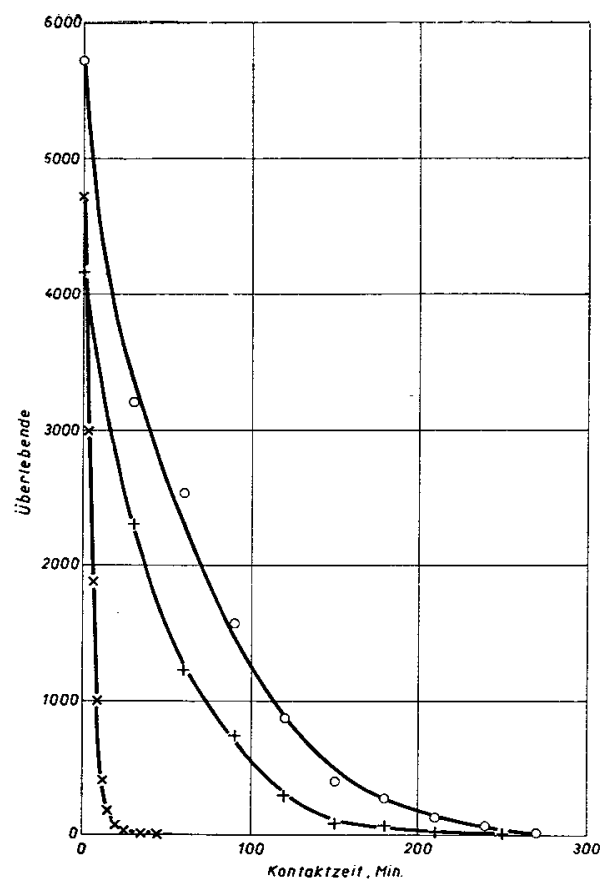

$a$

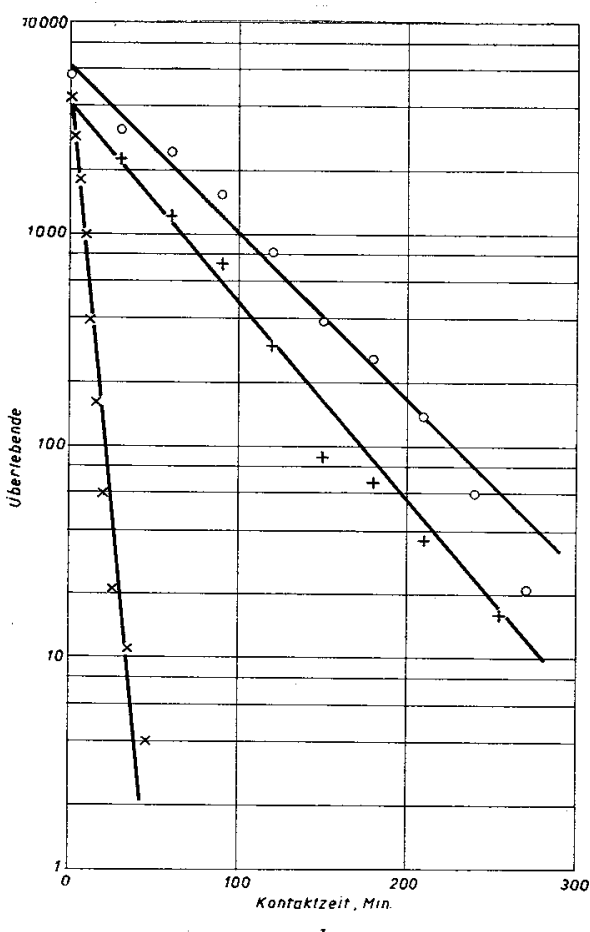

$b$

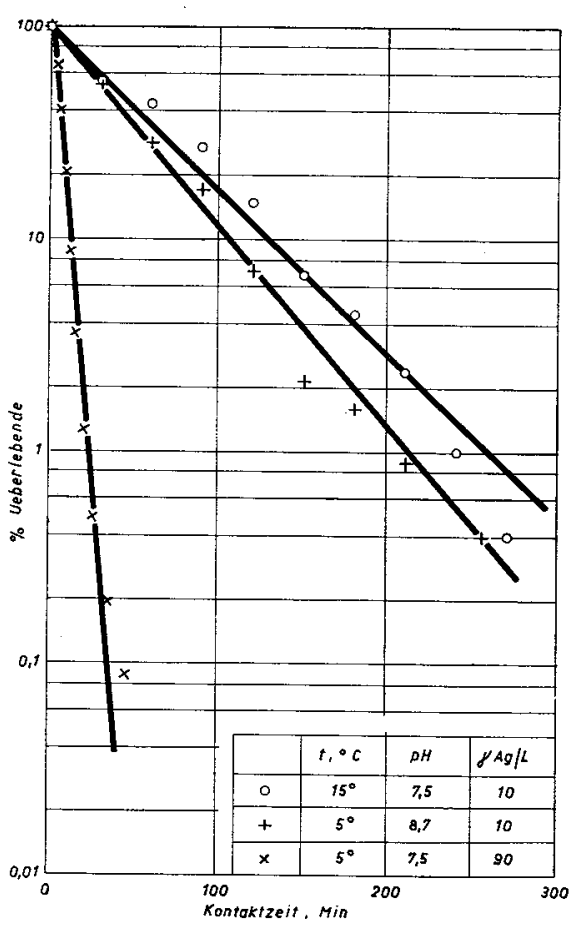

c

Abbildung 1

Absterbeordnung von ein-artigen Bakterienpopulationen in wässrigen Silberlösungen.

$a$ Überlebende und Kontaktzeit in absoluten Werten. $b$ log Überlebende in Funktion der Kontaktzeit (linear). $c$ Logarithmus der prozentual berech neten Überlebenden in Funktion der Kontaktzeit. Die Kurven in allen Abbildungen beziehen sich auf dieselben Experimente mit $E$. coli, Stamm I. 
exponentiellen Funktion (Abb. $\mathrm{I} a$ ), wie sie auch für viele andere Desinfektionsmittel bekannt ist. Trägt man in üblicher Weise die Logarithmen der überlebenden Keime in Beziehung zur Kontaktzeit auf (Abb. $\mathrm{r} b$ ), so findet man eine praktisch lineare Relation. Nicht lineare logarithmische Absterbekurven findet man immer, wenn:

a) Die Silberkonzentration im Laufe der Kontaktzeit durch Adsorption an die Gefässwände oder durch Umsetzungen, zum Beispiel mit Nährlösungsspuren, abnimmt. Die von OBERZILL [II] in dieser Hinsicht gemachten Beobachtungen stimmen mit unseren Ergebnissen durchaus überein.

b) Ein Gemisch verschiedener Keimarten der Silberwirkung ausgesetzt wird. Die entstehende, mehr oder weniger stark gekrümmte Kurve der Gesamtkeimzahlabnahme ist die Resultante der individuellen, linearen $\mathrm{Ab}$ sterbekurven (vgl. Abb. I $a$ und $\mathrm{i} b$ ).

\subsection{Bewertung der Absterbegeschwindigkeiten und der Streunngen der Absterbe- geschwindigkeiten von reinen Bakterienpopulationen}

Da aus praktischen Gründen die Anfangskeimzahl in den zu desinfizierenden Lösungen nicht genau gleich gross eingestellt werden kann, rechnet man bei quantitativen Vergleichen besser mit der prozentualen Abtötung als mit den absoluten Keimzahlen. Die linearen Absterbekurven von Abbildung I $c$ entsprechen der Beziehung

bzw.

$$
\begin{aligned}
& \log (1) \\
& 2-\log p_{\tau}=b \tau
\end{aligned}
$$

$p_{\tau}$ Prozent überlebende Keime nach der Kontaktzeit; $\tau$ Kontaktzeit; $b$ Konstante, Mass für die Absterbegeschwindigkeit.

Der Regressionskoeffizient $b$ der linearisierten Absterbekurve stellt die in vorstehendem Abschnitt erwähnte Grösse dar, die quantitative Vergleiche zwischen zwei unter verschiedenen äussern Bedingungen durchgeführten Desinfektionsversuchen gestattet. Dank der gewählten Transformation für die Zahl der überlebenden Keime $(2-\log p)$ besitzen sämtliche Absterbekurven denselben Ursprung, so dass sich eine stark vereinfachte statistische Behandlung von Serien von Absterbekurven ergibt, die miteinander verglichen werden sollen. Eine Regressionsgerade durch den Koordinatennullpunkt besitzt die allgemeine Form

$$
Y=b X
$$


Der Regressionskoeffizient $b$ ergibt sich für Geraden durch den Koordinatenursprung, die von Wertepaaren $x_{i} / y_{i}$ bestimmt sind, zu:

$$
b=\frac{S x_{i} y_{i}}{S x_{i}{ }^{2}},
$$

bzw. mit den Bezeichnungen der Desinfektionsexperimente:

$$
b=\frac{S\left[\tau_{i} \cdot\left(2-\log p_{\tau_{i}}\right)\right]}{S\left(\tau_{i}\right)^{2}}
$$

(Dimension von $b: \min ^{-1}$, wenn $\tau$ in Minuten gemessen wird).

Die benötigte Kontaktzeit $\tau$ für eine bestimmte Abtötungsrate (100-p\%) wird dann:

$$
\tau=\frac{(2-\log p)}{b}
$$

oder zum Beispiel für $b=0,0169 \mathrm{~min}^{-1}\left(30 \mu \mathrm{g} \mathrm{Ag}, 15^{\circ} \mathrm{C}, \mathrm{pH} 7,5, E\right.$. coli $)$ ergibt sich eine Kontaktzeit für

$$
\begin{aligned}
& 99 \% \text { Abtötung (1 \% Überlebende) }: \tau=\frac{2-\log 1}{0,0169}=\frac{2}{0,0169}=118 \mathrm{~min}, \\
& 99,99 \% \text { Abtötung }(0,01 \% \text { Überlebende }): \tau=\frac{2-\log 0,01}{0,0169}=\frac{4}{0,0169}=236 \mathrm{~min} .
\end{aligned}
$$

Wie aus Abbildung I a hervorgeht, streut in einem Absterbeversuch die Zahl der nach verschiedenen Kontaktzeiten überlebenden Keime oft ganz erheblich um die Regressionsgeraden $\log p=f(\tau)$, so dass die berechneten Werte von $b$ ebenfalls eine gewisse Variabilität besitzen. Aus einer grossen Zahl von Absterbekurven ergab sich, dass die Streuung von $b$ bei hohen Kontaktzeiten im allgemeinen höher ist als bei niederen. Eine proportionale Zunahme der Streuung mit der Kontaktzeit konnte aber nicht beobachtet werden. Es war deshalb leider nicht möglich, die Rechnungsmethoden für Regressionsgeraden durch den Koordinatenursprung, wie sie von $\mathrm{RAO}$ [13] angegeben wurden, anzuwenden, obschon besonders dieBerechnung der Streuung und der Vergleich verschiedener Regressionen damit einfacher geworden wäre.

Wir haben deshalb die Streuungen der Regressionskoeffizienten in üblicher Weise berechnet (vgl. zum Beispiel LINDER [9] oder BrownLEe [2]) und die Werte von $b$ bei Vergleichsversuchen mit dem $t$-Test auf Gleichartigkeit oder Verschiedenheit geprüft. Nach Berechnung der durchschnittlichen Streuung der Regressionskoeffizienten $s_{b}$ kann man bekannt- 
lich die Differenz zweier Regressionskoeffizienten $b^{\prime}$ und $b^{\prime \prime}$ auf ihre Signifikanz mit der Beziehung

$$
b^{\prime}-b^{\prime \prime}=t s_{b}
$$

prüfen, wobei $t$ bei $(N-2)$ Freiheitsgraden aufzuschlagen ist $\left.{ }^{3}\right)$.

\subsection{Einfluss einiger experimentell michtiger Faktoren auf die Abtötungs- geschpoindigkeit von E. coli}

Nachfolgend geben wir die Resultate von Experimenten bekannt, die im Sinne von Vorversuchen angestellt wurden, um diejenigen Einflüsse auf die Desinfektionswirkung von Silber kennenzulernen, die in der Praxis und vor allem bei quantitativen Versuchen berücksichtigt werden müssen.

2.33.I Einfluss pon $\mathrm{PO}_{4}^{-3}$ auf die Silberwirkung. Wie später gezeigt wird, übt der $\mathrm{pH}$-Wert einer silberhaltigen Lösung auf die Desinfektionswirkung des Silbers einen sehr grossen Einfluss aus. Es war daher unbedingt notwendig, die Versuchslösungen mittels Puffern genau auf einen gewollten $\mathrm{pH}-W e r t$ einzustellen. Im gewünschten pH-Bereich kommen - unter Berücksichtigung der übrigen Versuchsbedingungen - dafür lediglich Phosphatpuffer in Frage. Tabelle 2 und Abbildung 2 enthalten Angaben über den Einfluss des $\mathrm{PO}_{4}^{-3}$-Ions auf die Silberwirkung in destilliertem Wasser.

Tabelle 2

Einfluss von $\mathrm{PO}_{4}^{-3}$ auf die bakterizide Wirkung pon Silber (als $\mathrm{AgNO} \mathrm{O}_{3}$ )

\begin{tabular}{|c|c|c|c|c|}
\hline \multirow[t]{2}{*}{$\operatorname{mol~} \mathrm{PO}_{4}^{-3} / 1$} & \multicolumn{2}{|c|}{$\begin{array}{c}25 \mu g \mathrm{Ag}^{+} / 1 \mathrm{pH} 7,5 \\
25^{\circ} \mathrm{C}\end{array}$} & \multicolumn{2}{|c|}{$\underset{21^{\circ} \mathrm{C}}{60 \mu \mathrm{g} \mathrm{g}^{+} / 1} \mathrm{pH} 7,6$} \\
\hline & $b$ & $\begin{array}{c}\text { Minuten für } 99,9 \% \\
\text { Abtötung }\end{array}$ & $b$ & $\begin{array}{c}\text { Minuten für } 99,9 \% \\
\text { Abtötung }\end{array}$ \\
\hline $\begin{array}{l}0,001 \\
0,005 \\
0,01 \\
0,02 \\
0,03 \\
0,05\end{array}$ & $\begin{array}{l}0,0089 \\
0,0069 \\
0,0063 \\
0,0021 \\
0,0015\end{array}$ & $\begin{array}{r}335 \\
431 \\
476 \\
1400 \\
\\
1935\end{array}$ & $\begin{array}{l}0,0812 \\
0,0527 \\
0,0451 \\
\\
0,0063 \\
0,0049\end{array}$ & $\begin{array}{r}37 \\
57 \\
67 \\
\\
475 \\
612\end{array}$ \\
\hline
\end{tabular}

3) Wir möchten Herrn Prof. Dr. Linder für die Überprüfung der angewendeten statistischen Methoden bestens danken. 


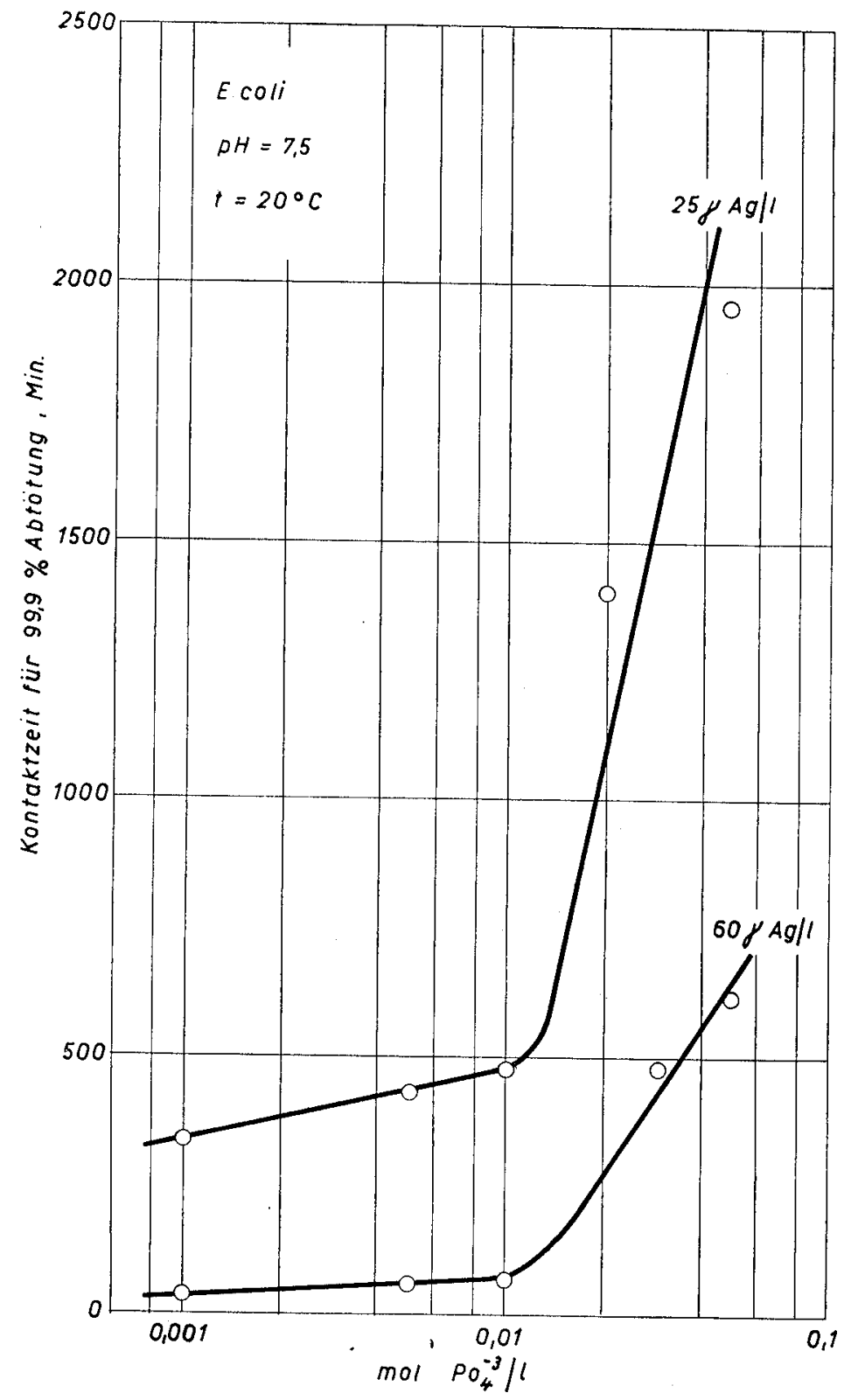

Abbildung 2

Einfluss des $\mathrm{PO}_{4}^{-3}$-Ions auf die bakterizide Wirkung von Silberlösungen mit konstantem Gehalt an Gesamt-Silber. 
Abbildung 2 zeigt, dass bei $\mathrm{PO}_{4}$-Konzentrationen über 0 , Or mol/l das Phosphat eine sehr starke Hemmung auf die Giftwirkung des Silbers ausübt. Auch unterhalb dieser Konzentrationsgrenze ist die Phosphathemmung noch deutlich bemerkbar (besonders bei geringen Silberkonzentrationen). Desinfektionsversuche in Gegenwart von 0,005 m Phosphatpuffer ergeben deshalb etwas geringere Abtötungsgeschwindigkeiten als in destilliertem Wasser mit gleichem $\mathrm{pH}$-Wert. Im Hinblick auf die grosse $\mathrm{pH}-\mathrm{Emp}-$ findlichkeit der Silberwirkung, die eine rigorose Einhaltung bestimmter $\mathrm{pH}$ Werte verlangt, musste aber dieser Nachteil der Phosphatpufferung in Kauf genommen werden.

2.33.2 Einfluss des Bakterienalters und der Bakteriendichte auf die Abtötungsgescbwindigkeit. Es ist bekannt, dass die Empfindlichkeit von Bakterien gegenüber Desinfektionsmitteln vom Bakterienalter abhängig ist. Gemäss Tabelle 3, welche parallele Abtötungsversuche mit dem für die meisten ausgewerteten Experimente verwendeten coli-Stamm enthält, geht folgendes hervor:

a) Die Abtötungsgeschwindigkeiten für Bakterien in der logarithmischen Wachstumsphase ( $16 \mathrm{~h}, 20 \mathrm{~h}$ ) sind beträchtlich kürzer als für dieselben Keime nach Erreichen der stationären Phase. Der Unterschied ist statistisch sehr gut gesichert.

Tabelle 3

Abbängigkeit der Ag-Empfundlicbkeit pon E. coli pom Entwicklungsstadium

\begin{tabular}{|c|c|c|c|c|c|c|}
\hline \multirow{2}{*}{ Vorkultur $^{\mathbf{1}}$ ) } & \multicolumn{6}{|c|}{$60 \mu \mathrm{g} \mathrm{Ag} / 1, t=21^{\circ}, 0,005 \mathrm{~m} \mathrm{PO}_{4}^{-3}$-Puffer, $\mathrm{pH} 7,5$} \\
\hline & 16 & 20 & 54 & 78 & 140 & $212 \mathrm{~h}$ \\
\hline $\begin{array}{l}\text { Geschwindigkeits- } \\
\left.\text { konstante } b^{2}\right) . \\
\text { Streuung von } b \\
\text { Kontaktzeit für } \\
99,9 \% \text { Abtötung } \\
\text { [min] . . . . . }\end{array}$ & $\begin{array}{r}0,06876 \\
\pm 0,00742 \\
\\
44\end{array}$ & $\begin{array}{c}0,07134 \\
\pm 0,00598 \\
42\end{array}$ & $\begin{array}{r}0,04998 \\
\pm 0,00788\end{array}$ & $\begin{array}{r}0,04977 \\
\pm 0,00611\end{array}$ & $\begin{array}{r}0,04609 \\
\pm 0,00718\end{array}$ & $\begin{array}{r}0,05273 \\
\pm 0,00427\end{array}$ \\
\hline $\begin{array}{l}\text { 1) Vorkultur: Milch } \\
\text { 2) Die Differenz zwi } \\
\text { 0,01795 beträgt. }\end{array}$ & $\begin{array}{l}\text { uckerbouill } \\
\text { schen zwei }\end{array}$ & $\begin{array}{l}\text { on, } 37^{\circ} \mathrm{C} \text {. } \\
b-\text { Werten ist }\end{array}$ & t gut gesich & $(P=0$ & 1), wenn sie & mindestens \\
\hline
\end{tabular}

b) Innerhalb der logarithmischen bzw. der stationären Entwicklungsphase sind mit dem vorliegenden Experiment keine statistisch gesicherten Empfindlichkeitsunterschiede nachweisbar. 
Da man in der Praxis der Wasserdesinfektion wohl immer mit Bakterienpopulationen in der stationären Entwicklungsphase zu tun haben wird, dehnten wir grundsätzlich bei coli-Versuchen die Vorkultur auf ungefähr $48 \mathrm{~h}$ aus.

Obschon theoretisch die Abtötungsgeschwindigkeit einer Bakterienaufschwemmung in einer Desinfektionslösung unabhängig von der Keimdichte sein sollte, beobachtet man häufig den gegenteiligen Effekt. Zum Teil mag dies daran liegen, dass bei hohen Keimdichten eine merkbare Konzentrationsverminderung der bakteriziden Substanz durch Adsorption an die Bakterien erfolgt (vom Übertragen von Nährlösungsspuren in die Desinfektionslösung durch ungenügendes Auswaschen der Bakterien sei abgesehen). Wie die Ergebnisse eines Kontrollversuches mit E. coli zeigen (Tab. 4), ist vorsichtshalber bei Vergleichsversuchen von Suspensionen mit möglichst gleichartiger Keimzahl auszugehen. Das Experiment zeigt nämlich, dass in dieser Versuchsreihe bei Überschreiten einer Anfangskeimzahl, die zwischen I 100 und $4800 \mathrm{Keimen} / \mathrm{cm}^{3}$ liegt, ein statistisch gesicherter Rückgang der Abtötungsgeschwindigkeit eintritt. Eine qualitativ analoge Beobachtung findet sich in der Arbeit von GoETz et al. [s].

Tabelle 4

Linfluss der Anfangskeimzabl in einer coli-Suspension auf dic Abtötungsgescboindigkeit durch Silber (als $\mathrm{AgNO}_{3}$ )

Bedingungen: $t=2 \mathrm{I}^{\circ}, \mathrm{pH}=7,5\left(\mathrm{PO}_{4}\right.$-Puffer $\left.0,005 \mathrm{~m}\right), 40 \mu \mathrm{g} \mathrm{Ag} \mathrm{g}^{+} / 1$

\begin{tabular}{|c|c|c|c|c|}
\hline & \multicolumn{4}{|c|}{ Anfangskeimzahl/ml } \\
\hline & $\begin{array}{c}A \\
8300\end{array}$ & $\begin{array}{c}B \\
4800\end{array}$ & $\begin{array}{c}C \\
1100\end{array}$ & $\begin{array}{c}D \\
260\end{array}$ \\
\hline $\begin{array}{l}b \\
\text { Streuung von } b \\
\tau_{99,9}[\mathrm{~min}]\end{array}$ & $\begin{aligned} 0,02006 \\
+0,00193 \\
\mathbf{1 5 0}\end{aligned}$ & $\begin{array}{r}0,01904 \\
+0,00112 \\
158\end{array}$ & $\begin{array}{c}0,02329 \\
\pm 0,00192 \\
129\end{array}$ & $\begin{aligned} 0,02420 \\
\pm \\
0,00194 \\
\mathbf{1 2 4}\end{aligned}$ \\
\hline $\begin{array}{l}\text { Signifikante Differenz } \\
P=0,05: \Delta b=0,0026 \mathrm{~b}\end{array}$ & $\begin{array}{l}\text { hen zwei } \\
\left.b_{A}=b_{B}\right)\end{array}$ & Werten & $=0,01:$ & $=0,0035$ \\
\hline
\end{tabular}

2.33.3 Einfluss der Silberberkunft und der $W$ asserqualität auf die bakterizide Wirkung einer Silberlösung. Für grundlegende Serienversuche schien uns die Verwendung eines leichtlöslichen Silbersalzes am einfachsten. Interessehalber prüften wir aber in einigen Experimenten auch die Frage, ob elektro- 
lytisch abgeschiedenes Silber annähernd gleiche bakterizide Eigenschaften aufweise wie zum Beispiel $\mathrm{Ag}^{+}$in Silbernitratlösungen. Für diese Versuche verwendeten wir einen sogenannten Elektro-Katadynapparat, den uns die Firma Katadyn AG, Wallisellen, freundlicherweise zur Verfuigung stellte. Gemäss Tabelle 5, welche die Ergebnisse der diesbezüglichen Kontrollversuche wiedergibt, hat man mit folgenden Tatsachen zu rechnen:

a) Vergleicht man Lösungen von elektrolytisch erzeugtem Silberion (Katadyn) und von Silbernitrat in Leitungsspasser, so kann kein Unterschied in der bakteriziden Wirkung beobachtet werden. Dieses Ergebnis stimmt mit den Angaben von HERRMANN [7] überein, der ebenfalls feststellte, dass Lösungen von $\mathrm{AgNO}_{3}, \mathrm{Ag}_{2} \mathrm{SO}_{4}$ und Ag-Acetat die gleiche Wirkung ausüben wie entsprechend konzentrierte, elektrolytisch hergestellteSilberlösungen.

b) In der Wirkung von Silbernitratlösungen zeigt sich in vorliegendem Experiment ein statistisch gesicherter Unterschied, indem $\mathrm{Ag}^{+}$in destilliertem Wasser rascher wirkt als in Leitungswasser. Wir werden in späteren Experimenten (vgl. Abschnitt 3.3) diese Beobachtung noch genauer darstellen.

Tabelle 5

Vergleich von $\mathrm{AgNO}_{3}$ und Katadyn-Silber

\begin{tabular}{|c|c|c|c|c|c|c|}
\hline \multirow{2}{*}{ Silberherkunft } & \multicolumn{6}{|c|}{$\left.50 \mu \mathrm{g} \mathrm{Ag} / 1, t=20^{\circ} \mathrm{C}, E . \cot ^{1}\right)$} \\
\hline & $\mathrm{AgNO}_{3}$ & $\mathrm{AgNO}_{3}$ & $\mathrm{AgNO}_{3}$ & App. I & App. I a & App. II \\
\hline Wasser & destilliert & $\mathrm{LW}(a)$ & $\mathrm{LW}(b)$ & $\mathrm{LW}$ & LW & $\left.\mathrm{LW}^{\mathbf{3}}\right)$ \\
\hline $\begin{array}{l}\text { Geschwindigkeits- } \\
\text { konstante } b^{2} \text { ) } \\
\text { Streuung von } b \pm \\
\text { Kontaktzeit für } \\
99,9 \% \text { Abtötung } \\
\text { [min] . . . . . }\end{array}$ & $\begin{array}{l}0,08386 \\
0,0218\end{array}$ & $\begin{array}{l}0,05705 \\
0,00530\end{array}$ & $\begin{array}{l}0,04760 \\
0,00485\end{array}$ & $\begin{array}{l}0,04539 \\
0,00530\end{array}$ & $\begin{array}{c}0,06405 \\
0,00627 \\
47 .\end{array}$ & $\begin{array}{c}0,06236 \\
0,00447 \\
48\end{array}$ \\
\hline $\begin{array}{l}\text { 1) Vorkultur } 48 \mathrm{~h} \\
\text { 2) Die Differenz } \\
\text { stens 0,0343 be } \\
\text { App. I } 3 \mathrm{Ag} \\
\text { App. I a } 3 \mathrm{Ag} \\
\text { App. II } 3 \mathrm{Ag} \\
\text { 3) Leitungswasser }\end{array}$ & $\begin{array}{l}\text { Milchzucker } \\
\text { ischen zwe } \\
\text { ägt. } \\
\text { noden; } \\
\text {-Anoden; } \\
\text { 3 C-Anod } \\
\text { Ca-Härte = }\end{array}$ & $\begin{array}{l}\text { ouillon } 37 \\
b \text {-Werten } \\
20 \text { frz. } \mathrm{H}^{\circ} \text {. }\end{array}$ & gut ges & $\operatorname{ert}(P$ & 1), wen & e minde- \\
\hline
\end{tabular}




\section{Der Einfluss einiger in der Praxis wichtiger Faktoren auf die Des- infektionswirkung von Silber}

Die nachstehend beschriebenen Versuchsergebnisse wurden in Experimenten gewonnen, die wir unter strenger Beachtung der in vorstehenden Abschnitten erwähnten methodischen Grundlagen vornahmen. Für alle zu prüfenden Faktoren wurden Reihenversuche angesetzt, die wir derart planten und durchführten, dass ihre statistische Auswertung möglich war.

\section{I Einfluss von Silberkonzentration, 'Temperatur und pH-Wert auf die Abtötungsgeschwindigkeit von E. coli}

Es handelt sich darum, sowohl den individuellen Einfluss der drei Faktoren als auch ihr Zusammenmirken auf die Abtötungsgeschwindigkeit von E. coli festzustellen. Als weiteres Ergebnis sollte aus den unter verschiedenen Bedingungen gewonnenen Geschwindigkeitskonstanten die Kontaktzeit für eine bestimmte Abtötungsrate in Funktion der Silberkonzentration sowie die Temperatur- und $\mathrm{pH}$-Abhängigkeit der Konzentrations-WirkungsFunktion ermittelt werden.

\section{I I Versuchsplan}

Zur Erreichung des Untersuchungszieles war es notwendig, Abtötungskurven für die Bakterien bei verschiedenen Wertestufen der drei Faktoren und allen Kombinationen dieser Stufen aufzunehmen. Aus ökonomischen und praktischen Gründen wurden folgende Abstufungen der Variablen gewählt:

Silberkonzentrationen: 10, 30, 90, 270 $\mu \mathrm{g} \mathrm{Ag} / \mathrm{l}$;

Temperaturen : $5^{\circ}, 15^{\circ}, 25^{\circ} \mathrm{C}$;

pH-Werte: 6,3, 7,5, 8,7.

Auf Grund der Erfahrungen bei den vielen Voruntersuchungen konnte davon ausgegangen werden, dass in einer Gruppe von Versuchen, die unter streng vergleichbaren Bedingungen durchgeführt wurden, die Streuungen der einzelnen Regressionen (\% Überlebende/Kontaktzeit) durch die gleichen Einwirkungen verursacht und normal verteilt sind. Damit waren die Voraussetzungen gegeben, das Experiment als sogenannten Faktorenversuch ohne Wiederholung der Einzelversuche durchzufïhren. Aus dem Programm der Faktorenversuche ergaben sich $4 \times 3 \times 3=36$ Einzel- 
experimente (Aufnahmen von Absterbekurven). Aus praktischen Gründen wurden drei Temperaturblöcke gebildet. Innerhalb der Temperaturblöcke erfolgten die Versuche in zufälliger Reihenfolge, und die drei Temperaturblöcke wurden ebenfalls in zufälliger Anordnung durchgeprüft.

\section{I2 Versuchsdurchführung}

Bakterien: E. coli, gleicher Stamm für alle Experimente. Vorkultur auf Milchzuckerbouillon bei $37^{\circ}, 30 \mathrm{~h}$, zweimaliges Auswaschen der Bakterien in Phosphatpuffer und Herstellung einer Impfsuspension mittels Verdünnung. Anfangskeimzahl in den Silberlösungen: $1700-3000$ pro $\mathrm{ml}$.

Silberlösungen: $\mathrm{AgNO}_{3}$-Lösungen in Phosphatpuffer $0,005 \mathrm{~mol} \mathrm{PO}_{4}^{-3}$, $\mathrm{pH}$ entsprechend Versuchsstufe.

Temperaturregelung: Die Desinfektionslösungen wurden in Wasserbäder der gewünschten Temperatur eingestellt $\left(t^{\circ} \pm 0,5^{\circ}\right)$.

Tabelle $6 \mathrm{a}$

Faktorenversuch, Zusammenstellung der beobacbteten Regressionsmerte

$$
b=\frac{2-\log p}{\tau}
$$

\begin{tabular}{|c|c|c|c|c|c|c|}
\hline \multirow{2}{*}{$t\left[{ }^{\circ} \mathrm{C}\right]$} & \multirow{2}{*}{$\mathrm{pH}$} & \multicolumn{4}{|c|}{ Silberkonzentration $\mu \mathrm{g} / 1$} & \multirow{2}{*}{$S$} \\
\hline & & 10 & 30 & 90 & 270 & \\
\hline \multirow[t]{2}{*}{$5^{\circ}$} & $\begin{array}{l}6,3 \\
7,5 \\
8,7 \\
\end{array}$ & $\begin{array}{l}0,00297 \\
0,00643 \\
0,01119 \\
\end{array}$ & $\begin{array}{l}0,00358 \\
0,01403 \\
0,02745 \\
\end{array}$ & $\begin{array}{l}0,01923 \\
0,03705 \\
0,05132 \\
\end{array}$ & $\begin{array}{l}0,05642 \\
0,08746 \\
0,16189 \\
\end{array}$ & \multirow[t]{2}{*}{$\begin{array}{l}0,08220 \\
0,14497 \\
0,25185\end{array}$} \\
\hline & $S$ & 0,02059 & 0,04506 & 0,10760 & 0,30577 & \\
\hline \multirow[t]{2}{*}{$15^{\circ}$} & $\begin{array}{l}6,3 \\
7,5 \\
8,7 \\
\end{array}$ & $\begin{array}{l}0,00361 \\
0,00950 \\
0,01387 \\
\end{array}$ & $\begin{array}{l}0,00873 \\
0,01693 \\
0,03002 \\
\end{array}$ & $\begin{array}{l}0,02076 \\
0,04761 \\
0,07783 \\
\end{array}$ & $\begin{array}{l}0,09344 \\
0,13980 \\
0,22089 \\
\end{array}$ & \multirow[t]{2}{*}{$\begin{array}{l}0,12654 \\
0,21384 \\
0,34261\end{array}$} \\
\hline & $S$ & 0,02698 & 0,05568 & 0,14620 & 0,45413 & \\
\hline \multirow[t]{2}{*}{$25^{\circ}$} & $\begin{array}{l}6,3 \\
7,5 \\
8,3\end{array}$ & $\begin{array}{l}0,00248 \\
0,00709 \\
0,01475\end{array}$ & $\begin{array}{l}0,01977 \\
0,03487 \\
0,07438 \\
\end{array}$ & $\begin{array}{l}0,04375 \\
0,09277 \\
0,15170 \\
\end{array}$ & $\begin{array}{l}0,15051 \\
0,23363 \\
0,36898\end{array}$ & \multirow[t]{2}{*}{$\begin{array}{l}0,21651 \\
0,36836 \\
0,60981\end{array}$} \\
\hline & $S$ & 0,02432 & 0,12902 & 0,28822 & 0,75312 & \\
\hline \multirow[t]{2}{*}{$S$} & $\begin{array}{l}6,3 \\
7,5 \\
8,9 \\
\end{array}$ & $\begin{array}{l}0,00906 \\
0,02302 \\
0,03981 \\
\end{array}$ & $\begin{array}{l}0,03208 \\
0,06583 \\
0,13185 \\
\end{array}$ & $\begin{array}{l}0,08374 \\
0,17743 \\
0,28085 \\
\end{array}$ & $\begin{array}{l}0,30037 \\
0,46089 \\
0,75176 \\
\end{array}$ & \multirow[t]{2}{*}{$\begin{array}{l}0,42525 \\
0,72717 \\
1,20427\end{array}$} \\
\hline & Total & 0,07189 & 0,22976 & 0,54202 & 1,51302 & \\
\hline
\end{tabular}


Tabelle $6 \mathrm{~b}$

Streungszerlegung für die Werte in Tabelle 6 a

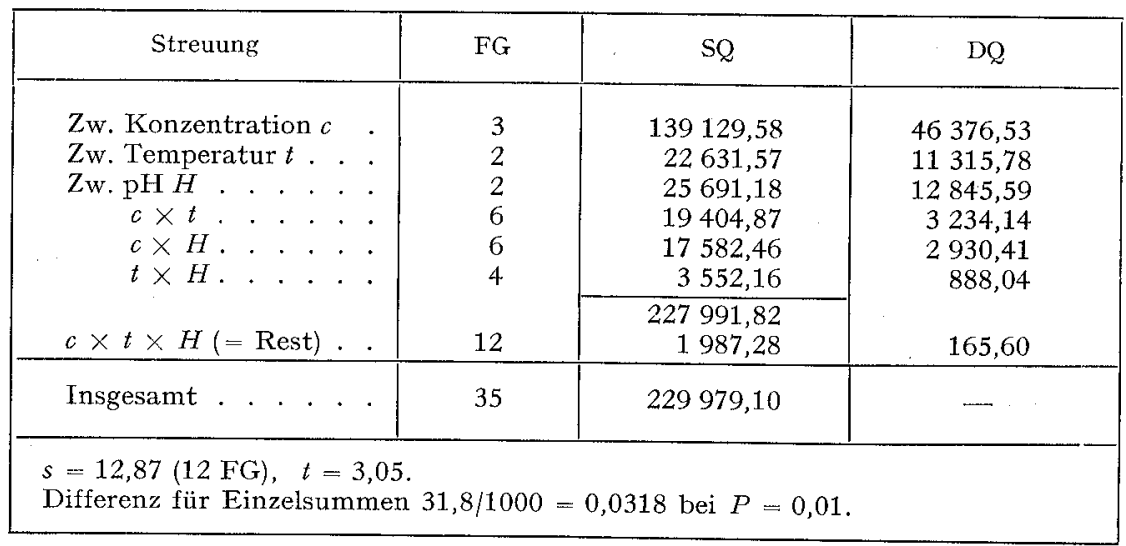

Tabelle 7

Faktorenpersuch, Kontaktzeiten in Minuten für 99,9\% Abtötung von E. coli (auf Minuten gerundet)

\begin{tabular}{|c|c|c|c|c|c|}
\hline \multirow{3}{*}{$\begin{array}{c}\text { Temperatur } \\
{\left[{ }^{\circ} \mathrm{C}\right]}\end{array}$} & \multirow{3}{*}{$\mathrm{pH}$} & \multicolumn{4}{|c|}{ Silberkonzentration $\mu g \mathrm{Ag} / 1$} \\
\hline & & 10 & 30 & 90 & 270 \\
\hline & & \multicolumn{4}{|c|}{ Minuten } \\
\hline $5^{\circ}$ & $\begin{array}{l}6,3 \\
7,5 \\
8,7\end{array}$ & $\begin{array}{r}1010 \\
466 \\
268\end{array}$ & $\begin{array}{l}837 \\
214 \\
109\end{array}$ & $\begin{array}{r}156 \\
81 \\
58\end{array}$ & $\begin{array}{l}53 \\
34 \\
18\end{array}$ \\
\hline $15^{\circ}$ & $\begin{array}{l}6,3 \\
7,5 \\
8,7\end{array}$ & $\begin{array}{l}831 \\
316 \\
216\end{array}$ & $\begin{array}{l}344 \\
177 \\
100\end{array}$ & $\begin{array}{r}144 \\
63 \\
38\end{array}$ & $\begin{array}{l}32 \\
21 \\
13\end{array}$ \\
\hline $25^{\circ}$ & $\begin{array}{l}6,3 \\
7,5 \\
8,7\end{array}$ & $\begin{array}{r}1210 \\
423 \\
203\end{array}$ & $\begin{array}{r}152 \\
86 \\
40\end{array}$ & $\begin{array}{l}68 \\
32 \\
20\end{array}$ & $\begin{array}{r}20 \\
13 \\
8\end{array}$ \\
\hline
\end{tabular}

Die auf den gewünschten $\mathrm{pH}$-Wert gepufferten Silberlösungen wurden nach Temperierung mit I $\mathrm{ml}$ der Bakteriensuspension beimpft. Nach Ablauf jeder Kontaktperiode wurden zwei Proben zu je I ml auspipettiert und zu Glukose-Pepton-Agarplatten verarbeitet. Die unmittelbar nach Impfung der Silberlösungen erhobenen Proben (Kontaktzeit o min) dienten zur ge- 
nauen Feststellung der Anfangskeimzahl $(p=100 \%)$. Die Glukose-AgarPlatten wurden bei $37^{\circ}$ bebrütet und nach $24 \mathrm{~h}$ ausgezählt. Für die Berechnung der Resultate wurden jeweils die Summen der Doppelproben verwendet.

Von allen Abtötungsreihen wurden zunächst die Prozente der überlebenden Keime ( $p$ ) berechnet und auf semi-logarithmischem Papier zur Feststellung der Linearität der Absterbefunktion $\log p /$ Kontaktzeit aufgetragen. Sämtliche 36 Abtötungsfunktionen erwiesen sich als sehr gut linear, so dass die in Abschnitt 2.32 dargestellten Berechnungsverfahren ohne weiteres angewendet werden konnten.

\subsection{Versuchsergebnisse}

In Tabelle $6 a$ sind die Regressionskoeffizienten $b=(2-\log p) / \tau$ der einzelnen Absterbefunktionen zusammengestellt. Dank der gewählten Versuchsanordnung kann eine Streuungszerlegung für diese Werte vorgenommen werden, die sich in Tabelle $6 \mathrm{~b}$ findet. Für praktische Zwecke haben wir die Kontaktzeiten für $99,9 \%$ Abtötung aus den $b$-Werten von Tabelle 6 a berechnet und in Tabelle 7 zusammengefasst. Mit Hilfe dieser Angaben lässt sich nun die gesuchte Konzentrations-Wirkungs-Funktion des Silbers unter den gewählten äusseren Bedingungen aufzeichnen (Abb.3).

\section{I4 Auswertung der Beobacbtungen}

Eine Durchsicht der Werte für die Geschwindigkeitskonstanten der coliAbtötung in Tabelle 6 a zeigt sofort, dass sowohl die Konzentrationen als auch die Temperatur und die $\mathrm{pH}$-Werte der Silberlösungen einen starken Einfluss ausüben. Die Streuungszerlegung (Tab. 6b) ergibt, dass diese Hauptwirkungen der drei Faktoren statistisch sehr gut gesichert sind. Es kann allerdings nicht übersehen werden, dass trotz sorgfältiger Beachtung konstanter Versuchsbedingungen erhebliche Unregelmässigkeiten bei der systematischen Gruppierung der Werte von $b$ (und dementsprechend auch von $\tau_{99,9}$ in Tabelle 7 ) auftreten. Dies betrifft zur Hauptsache nur die Versuchsreihe mit der Silberkonzentration von Io $\mu \mathrm{g} A g / 1$. Da in diesem Konzentrationsbereich schon kleinste Konzentrationsverschiebungen, die fast unvermeidlich sind, einen grossen Einfluss auf die Abtötungsgeschwindigkeit ausüben (vgl. auch Abb. 3), haben wir diese Reihe bei der späteren Auswertung der $\mathrm{pH}$ - und Temperaturfunktion weggelassen. 


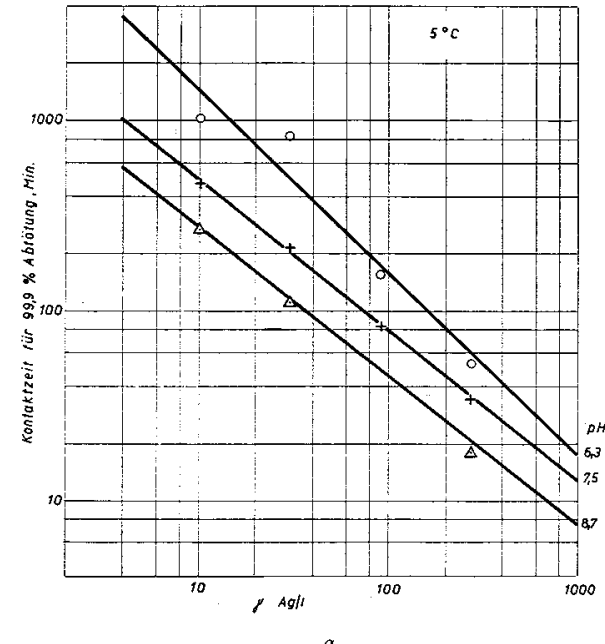

$a$

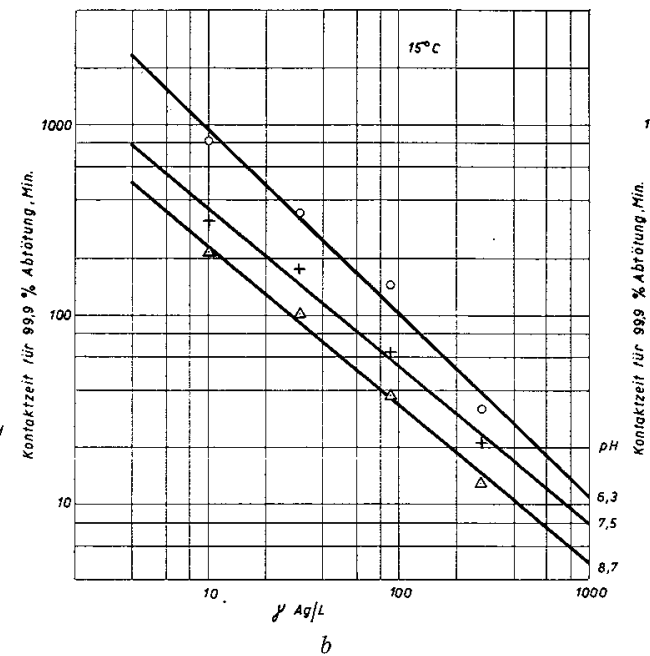

Abbildung 3

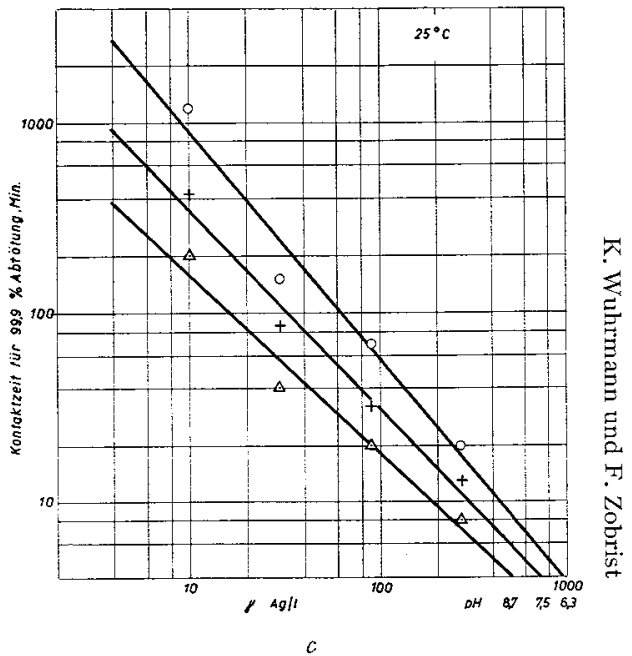

Konzentrations-Wirkungs-Kurven von Silber auf E. coli I in Funktion der Temperatur und des pH-Wertes der Silberlösung. $a$ Temperatur $=5^{\circ} \mathrm{C}, b$ Temperatur $=15^{\circ} \mathrm{C}, c$ Temperatur $=25^{\circ} \mathrm{C}$. 
Die Streuungsanalyse erweist ferner, dass sämtliche $W$ echselwirkungen der gepriuften Variablen ebenfalls statistisch gesichert sind (auch wenn die IO- $\mu$ g-Reihe weggelassen wird), oder mit anderen Worten:

Der Temperatur- und pH-Einfluss auf die absoluten Werte der Abtötungsgeschwindigkeit von $E$. coli ist bei unterschiedlichen Silberkonzentrationen verschieden gross;

der Einfluss des $\mathrm{pH}$-Wertes wechselt mit der'Temperatur und umgekehrt.

Trotz der vorstehend erwähnten Streuungen der $b$-Werte sind diese Wechselwirkungen so gut gesichert, dass sie theoretisch und praktisch eine grosse Bedeutung besitzen, und wir haben versucht, ein quantitatives Mass für den Einfluss dieser beiden Faktoren zu gewinnen.

Der Temperatureinfluss auf die Geschwindigkeit biologischer Vorgänge ist bekanntlich in sehr vielen Fällen innerhalb eines gewissen Temperaturbereiches annähernd konstant, und wenn es sich um einen Vorgang handelt, der nach dem Schema einer Reaktion erster Ordnung verläuft, gilt angenähert:

$$
\log \frac{k_{1}^{\prime}}{k_{1}^{\prime \prime}}=q\left(t_{2}-t_{1}\right)
$$

das heisst, der Logarithmus des Verhältnisses der Geschwindigkeitskonstanten $k_{1}$ bei zwei Temperaturen ist der Temperaturdifferenz $\left(t_{2}-t_{1}\right)$ proportional. Trägt man die in Tabelle 8 a zusammengestellten Logarithmen der $b$-Werte graphisch in Funktion der Temperatur auf, so ist die Linearität der Beziehung unzweifelhaft (vgl. Abb. 4a), obschon ebenfalls nicht unbeträchtliche Variabilitäten vorkommen.

Eigenartigerweise findet man nun für die Beziehung von $b \mathrm{zu}$ den $p H-$ Werten genau den gleichen Zusammenhang wie bei der Temperatur (vgl. Abb. 4 b).

Aus den beiden Abbildungen $4 a$ und $4 b$ geht hervor, dass der Gradient des Temperatur- bzw. pH-Einflusses im Beobachtungsbereich offenbar praktisch unabhängig von der Silberkonzentration ist. Wir haben deshalb von den Logarithmen der $b$-Werte ebenfalls eine Streuungsanalyse vorgenommen (Tab. $8 \mathrm{~b}$ ), wobei die Io- $\mu \mathrm{g}$-Reihe wegen ihrer Unregelmässigkeit nicht berücksichtigt wurde. Das Resultat ist folgendes:

Erwartungsgemäss sind die Hauptwirkungen der drei Faktoren Silberkonzentration, Temperatur und $\mathrm{pH}$ sehr gut gesichert.

Sämtliche Wecbselmirkungen zwischen den Faktoren sind statistisch nicht mebr signifikant, oder mit anderen Worten: Weder Silberkonzentration noch $\mathrm{pH}$-Wert beeinflussen den Gradienten der Temperaturwirkung im Bereich 
Tabelle $8 \mathrm{a}$

Faktorenversucb, Logaritbmus der Regressionswerte

$$
b=\frac{2-\log p}{\tau} \cdot 1000
$$

\begin{tabular}{|c|c|c|c|c|c|}
\hline \multirow{2}{*}{$t$} & \multirow{2}{*}{$\mathrm{pH}$} & \multicolumn{3}{|c|}{ Silberkonzentration $\mu g / 1$} & \multirow{2}{*}{ Summe } \\
\hline & & 30 & 90 & 270 & \\
\hline $5^{\circ}$ & $\begin{array}{c}6,3 \\
7,5 \\
8,7 \\
S\end{array}$ & $\begin{array}{l}0,5538 \\
1,1470 \\
1,4385 \\
3,1393\end{array}$ & $\begin{array}{l}1,2839 \\
1,5687 \\
1,7102 \\
4,5628\end{array}$ & $\begin{array}{l}1,7514 \\
1,9418 \\
2,2092 \\
5,9024\end{array}$ & $\begin{array}{r}3,5891 \\
4,6575 \\
5,3579 \\
13,6045\end{array}$ \\
\hline $15^{\circ}$ & $\begin{array}{c}6,3 \\
7,5 \\
8,7 \\
S\end{array}$ & $\begin{array}{l}0,9410 \\
1,2286 \\
1,4774 \\
3,6470\end{array}$ & $\begin{array}{l}1,3122 \\
1,6777 \\
1,8911 \\
4,8860\end{array}$ & $\begin{array}{l}1,9704 \\
2,1455 \\
2,3441 \\
6,4600\end{array}$ & $\begin{array}{r}4,2286 \\
5,0518 \\
5,7126 \\
14,9930\end{array}$ \\
\hline $25^{\circ}$ & $\begin{array}{c}6,3 \\
7,5 \\
8,7 \\
S\end{array}$ & $\begin{array}{l}1,2960 \\
1,5424 \\
1,8714 \\
4,7098\end{array}$ & $\begin{array}{l}1,6408 \\
1,9674 \\
2,1809 \\
5,7891\end{array}$ & $\begin{array}{l}2,1775 \\
2,3685 \\
2,5670 \\
7,1130\end{array}$ & $\begin{array}{r}5,1143 \\
5,8783 \\
6,6193 \\
17,6119\end{array}$ \\
\hline Summe & $\begin{array}{l}6,3 \\
7,5 \\
8,7\end{array}$ & $\begin{array}{l}2,7908 \\
3,9180 \\
4,7873\end{array}$ & $\begin{array}{l}4,2419 \\
5,2138 \\
5,7822\end{array}$ & $\begin{array}{l}5,8993 \\
6,4558 \\
7,1203\end{array}$ & $\begin{array}{l}12,9320 \\
15,5876 \\
17,6898\end{array}$ \\
\hline Total & & 11,4961 & 15,2379 & 19,4754 & 46,2094 \\
\hline
\end{tabular}

Tabelle $8 \mathrm{~b}$

Streuungszerlegung der Werte von Tabelle 8 a

\begin{tabular}{|c|c|c|c|}
\hline Streuung & $\mathrm{FG}$ & SQ & $\mathrm{DQ}$ \\
\hline 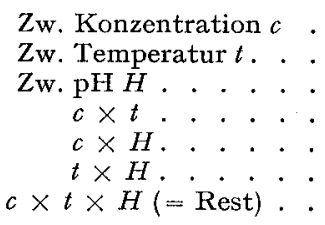 & $\begin{array}{l}2 \\
2 \\
2 \\
4 \\
4 \\
4 \\
8\end{array}$ & $\begin{array}{l}3,54173 \\
0,92022 \\
1,26327 \\
0,02206 \\
0,05835 \\
0,01174 \\
0,04608\end{array}$ & $\begin{array}{l}1,77086 \\
0,46011 \\
0,63163 \\
0,00551 \\
0,01459 \\
0,00293 \\
0,00576\end{array}$ \\
\hline Insgesamt . . . . . . & 26 & 5,86345 & - \\
\hline
\end{tabular}




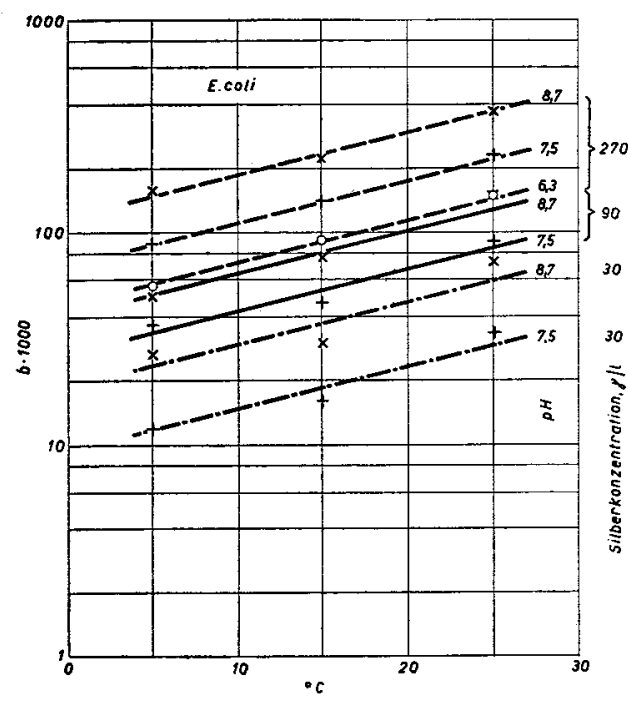

a

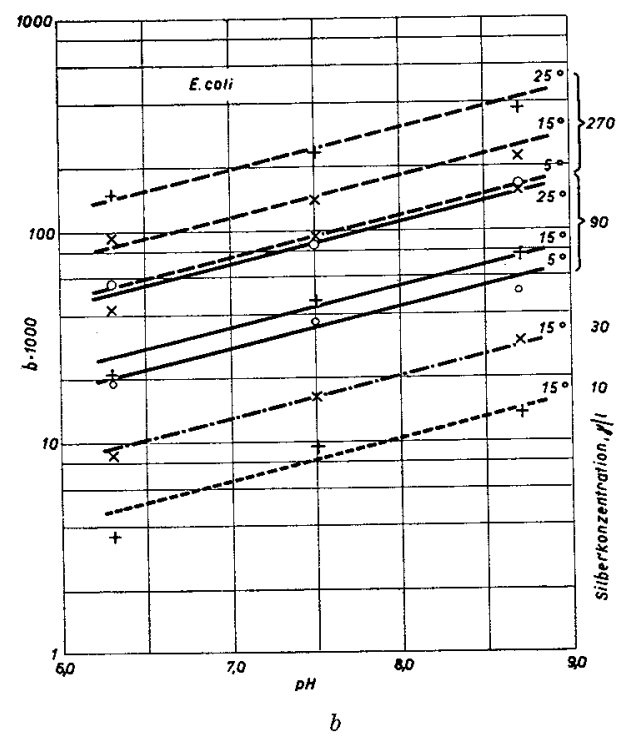

Abbildung 4

Beispiele für den linearen Zusammenhang zwischen den Logarithmen der Absterbegeschwindigkeiten und dem $\mathrm{pH}$-Wert ( $a$ ) bzw, dẹr Temperatur $(b)$ der Silberlösung. Ordinațen: Werte von $1000 \times b$ aus Tabelle 6 a. 
von $5^{-25^{\circ}}$. Desgleichen ist der Gradient des pH-Einflusses von der Temperatur und der Silberkonzentration unabhängig.

Aus diesen Feststellungen ergibt sich automatisch, dass die Temperaturbzw. pH-Gradienten aller Versuchsreihen derselben Grundgesamtheit angehören, und die entsprechenden linearen Funktionen (Abb. 4a,b) also parallel sind. Es kann aus diesem Grunde deshalb ein gemeinsamer Temperatur- bzw. pH-Gradient (zum Beispiel aus der Summe aller Einzelwerte von $\log b$ ) ermittelt werden. Die Durchführung dieser Berechnung ergibt:

a) Für den Temperatur-Gradienten:

$$
\frac{1}{t_{2}-t_{1}} \log \frac{\bar{b}_{2}}{\bar{b}_{1}}=0,02035
$$

bzw. für $t_{2}-t_{1}=10^{\circ} \mathrm{C}: \Theta_{10}=$ num. $0,2035=7,59$, gültig im Bereich von $5-25^{\circ}$, $\mathrm{pH} 6,3-8,7$ und $30-270 \mu \mathrm{g} \mathrm{Ag} / \mathrm{l}$.

b) Für den $p H$-Gradienten:

$$
\frac{1}{\mathrm{pH}_{2}-\mathrm{pH}_{1}} \log \frac{\bar{b}_{2}}{\bar{b}_{1}}=0,1895
$$

bzw. für $\mathrm{pH}_{2}-\mathrm{pH}_{1}=1$ Einheit: $\Pi_{1}=$ num. $0,1895=1,55$, gültig im Bereich von $\mathrm{pH} 6,3-8,7,5^{\circ}-25^{\circ}$ und $30-270 \mu \mathrm{g} \mathrm{Ag} / \mathrm{l}$.

Man ist berechtigt, die beiden Gradienten auch für den Konzentrationsbereich unterhalb $30 \mu \mathrm{g} A g / \mathrm{l}$ als gültig zu betrachten. Zusammenfassend ergibt sich also:

a) Die Steigerung der Wassertemperatur um $10^{\circ}$ beschleunigt die Silberwirkung auf $E$. coli um das $\mathrm{I}$,6fache, unabhängig von $\mathrm{pH}$-Wert und Konzentration der Silberlösung.

b) Die Steigerung des $\mathrm{pH}$-Wertes des Wassers um eine $\mathrm{pH}$-Einheit ( $\mathrm{Ab}$ nahme der $\mathrm{H}^{+}$-Konzentration um das Iofache) beschleunigt die Silberwirkung um das I, 55 fache, unabhängig von der Temperatur und der Konzentration der Ag-Lösung.

In der Literatur findet sich unseres Wissens nur eine einzige Angabe über Temperaturkoeffizienten der bakteriziden Wirkung von Silber bei CHICK [20]. Diese Autorin arbeitete mit B. paratyphosum und fand Werte für $\Theta_{10}$ von 2,8 bis 3,0 . Die gegenüber unseren Beobachtungen fast doppelt so hohen Werte können nicht auf Verhaltensunterschiede von E. coli bezw B. paratyphosum zurückgeführt werden. Wir glauben, dass die Angaben von 
CHICK viel zu hoch ausgefallen sind, und zwar wegen der verwendeten Mess- und Berechnungsmethoden.

Unsere Beobachtungen über den pH-Einfluss auf die Abtötungsgeschwindigkeit von $E$. coli in wässrigen Lösungen stehen in Widerspruch zu den Feststellungen von GoeTz et al. [5]. Diese Autoren gaben an, dass bei pHWerten zwischen 7,0 und 8,0 ein Optimum der Giftwirkung von Silberlösungen liege. Diese Beobachtung wurde jedoch bei Anwendung von Silber in Näbrlösungen gemacht. Sie gibt deshalb mit aller Wahrscheinlichkeit nicht die Wechselwirkung $\mathrm{Ag}^{+}-\mathrm{H}^{+}$-Organismus, sondern in erster Linie diejenige von $\mathrm{Ag}^{+}-\mathrm{H}^{+}-\mathrm{Näh}$ rbodensubstanz wieder und ist deshalb toxikologisch uninteressant.

\subsection{Einfluss von Chloriden auf die Silberwirkung}

Erwartungsgemäss tritt durch die Gegenwart von Chloridionen in Wasser eine Hemmung der Silberwirkung auf (Bildung von $\mathrm{AgCl}$ und eventuell auch anderer Chlor-Silber-Verbindungen). Über den Umfang der Verlängerung der Kontaktzeit in Funktion des Chloridgehaltes (Zusatz als $\mathrm{NaCl}$ ) einer Silberlösung orientiert Abbildung 5. Die Darstellung enthält die in Funktion der Chloridkonzentration effektiv vorhandene Konzentration an Silberionen, wenn ein Äquivalent von $60 \mu \mathrm{g} \mathrm{Ag} / 1$ zur Chloridlösung gegeben wird (Kurve $\mathrm{Ag}^{+}$). Ferner sind die in den chloridhaltigen $A g$-Lösungen effektiv beobachteten Abtötungszeiten von $E$. coli für $99,9 \%$ Abtötung eingetragen (Kurve $\tau_{\mathrm{Cl}}$ ). Als dritte Kurve sind die Abtötungszeiten für E. coli angegeben, welche der effektiv in der Lösung vorhandenen Konzentration an Silberionen entsprechen (Kurve $\tau_{0}$ ). Die Kurve $\mathrm{Ag}^{+}$wurde aus dem Löslichkeitsprodukt von $\mathrm{AgCl}$ bei den gewählten Versuchsbedingungen berechnet (Handbook of Chemistry, 1955). Abbildung 5 zeigt mit aller Deutlichkeit, dass durch die Gegenwart von Chloridionen in einer Silberlösung die Giftwirkung des vorhandenen Silbers beträchtlich vermindert wird (Kontaktzeit für 99,9\% Abtötung bei $\mathrm{Cl}^{--}=0 \mathrm{mg} / \mathrm{l}: 40 \mathrm{~min}$, bei $\mathrm{Cl}^{-}=500 \mathrm{mg} / \mathrm{l}: 270 \mathrm{~min}$ ). Die Zunahme der Kontaktzeit ist aber viel geringer, als sie aus der Abnahme der freien Silberionen in der Lösung erwartet werden müsste. Vorbehältlich eingehenderer Untersuchungen muss man vorläufig annehmen, dass die in den verdünnten Lösungen sich bildenden Partikel von Chlor-Silber-Verbindungen wenigstens teilweise noch bakterizid wirken können. Unsere Untersuchungen bestätigen in qualitativer Hinsicht die Beobachtungen von HerrmanN [7]. Er stellte allerdings im Bereiche von o-50 $\mathrm{mg} \mathrm{Cl}^{-} / 1$ einen 


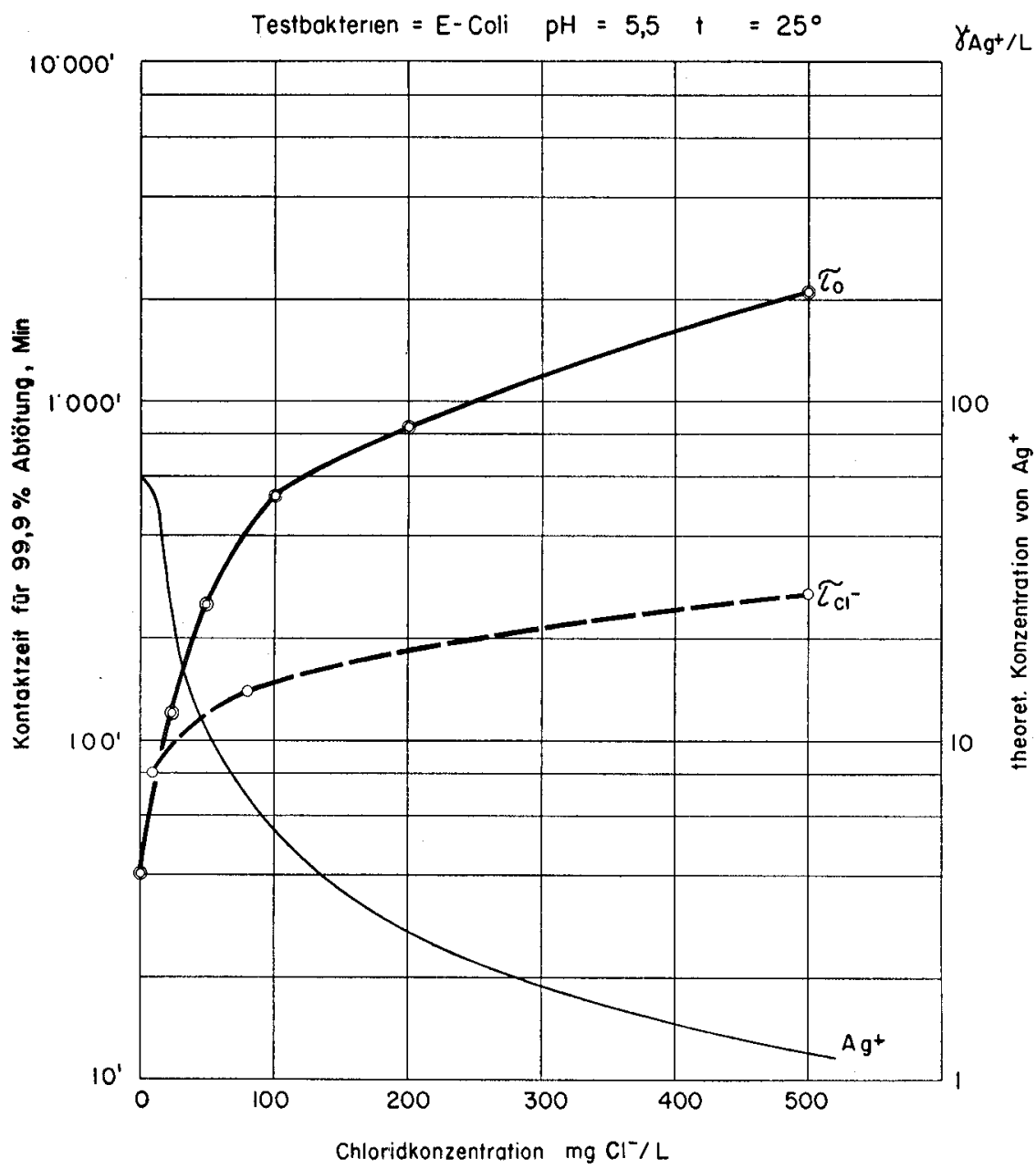

Abbildung 5

Einfluss von Chloridionen auf die bakterizide Wirkung von wässrigen Silberlösungen. Kurve $\mathrm{Ag}^{+}$: Konzentration von Silberionen in Funktion der Konzentration von Chloridionen (Silberzusatz entspricht $60 \mu \mathrm{g} \mathrm{Ag} / 1)$; Kurve $\tau_{0}$ : Abtötungszeit für $E$. coli, die dem von der Kurve Ag ${ }^{\dagger}$ angegebenen Gehalt der Lösung an Silberionen entspricht (gemäss Berechnung aus Konzentrations-Wirkungs-Kurve von Abbildung 3); Kurve $\tau_{\mathrm{CI}}$ : effektiv beobachtete Abtötungszeiten in chloridhaltigen Lösungen mit einem totalen Ag-Gehalt von $60 \mu \mathrm{g} / \mathrm{l}$. 
wesentlich stärkeren Anstieg der Kontaktzeiten fest als wir. Bei $\mathrm{Cl}^{--}$ Konzentrationen von $50-5000 \mathrm{mg} \mathrm{Cl} / 1$ fand er aber ebenfalls eine verhältnismässig langsame Abnahme der Abtötungsgeschwindigkeiten, die mit der berechenbaren Abnahme der $\mathrm{Ag}^{+}-$Konzentration in keinem Verhältnis stand.

Auf Grund der bisherigen Beobachtungen gelangt man zur Schlussfolgerung, dass die Chloridkonzentration eines Wassers bei der Festlegung der Kontaktzeiten unbedingt zu berücksichtigen ist. Ihr Einfluss entspricht aber nicht der theoretisch errechenbaren Abnahme der $\mathrm{Ag}^{+}-$Konzentration, sondern ist viel geringer. Bei Aktivierungen von $60 \mu \mathrm{g} \mathrm{Ag} / \mathrm{l}$ verursachte in unseren Versuchen eine Konzentration von to $\mathrm{mg} \mathrm{Cl}^{-} / 1$ eine Erhöhung der Kontaktzeit um rund $25 \%$, bei $100 \mathrm{mg} \mathrm{Cl}-/ 1$ um rund $70 \%$ beim untersuchten coli-Stamm.

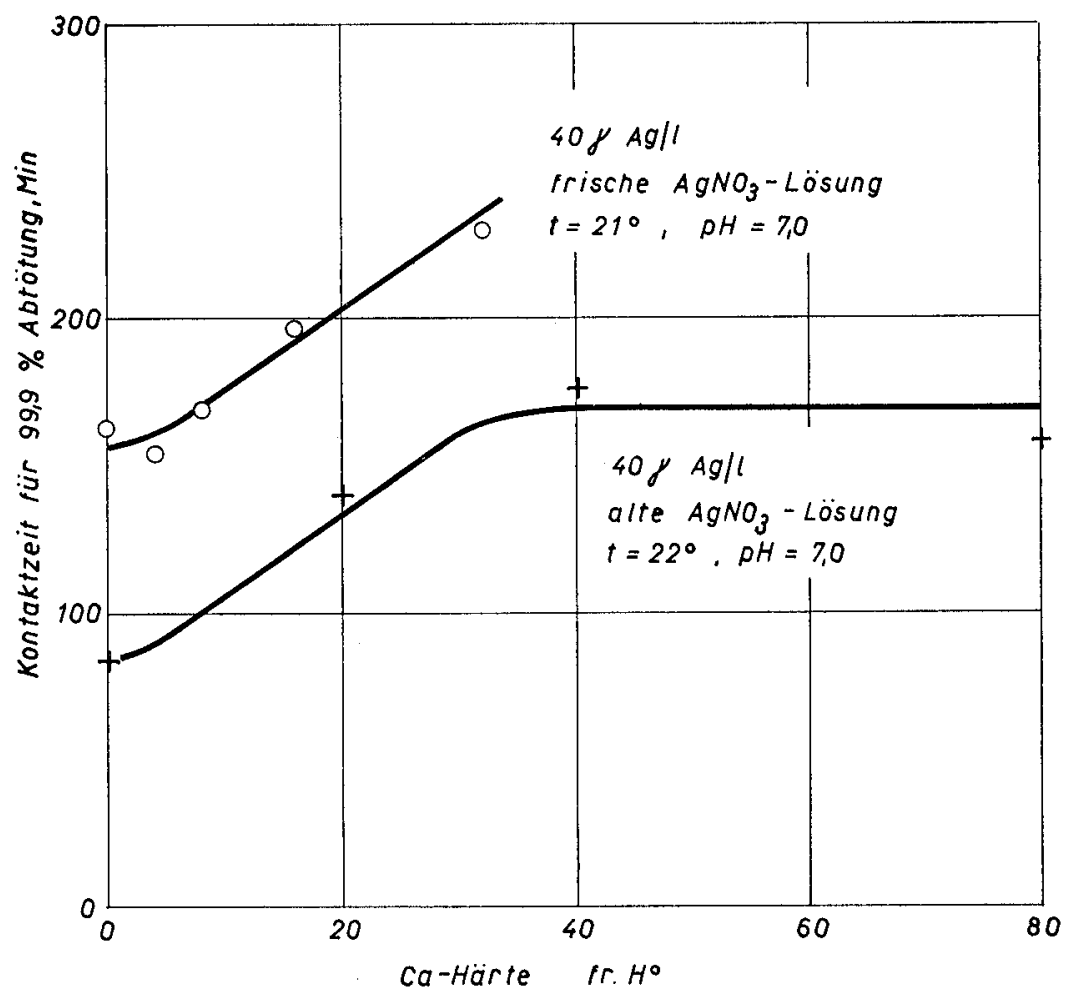

Abbildung 6

Einfluss der Kalziumhärte des Wassers auf die bakterizide Wirkung von Silberlösungen. Beachte die Wirkungsdifferenz der beiden verschieden alten Silberlösungen (Versuch mit $E$. coli I). 


\subsection{Einfluss der Wasserhärte}

Wir haben bisher lediglich geprüft, wie weit Calciumionen die Wirkung einer Silberlösung beeinflussen, da ihnen hinsichtlich Einwirkung auf die Permeabilitätsverhältnisse von Zellen die grösste Bedeutung unter den Härtebildnern zukommt. Die Versuche mussten wegen der sich anfänglich stark widersprechenden Resultate mehrmals wiederholt werden. Wir haben in Abbildung 6 die Ergebnisse von zwei massgebenden Experimenten zusammengestellt, die grundsätzlich dasselbe zeigen:

Die Wirkung einer Silberlösung wird von Calciumionen merklich verlangsamt, und zwar speziell im Bereich der Calciumhärtegrade, die sich normalerweise bei Trinkwässern finden (Io-40 fr. Härtegrade). Der Mechanismus dieser Ca-Wirkung ist noch unabgeklärt, doch scheint uns ein Permeabilitätsantagonismus zwischen $\mathrm{Ca}^{+2}$ und $\mathrm{Ag}^{+}$vorläufig als plausibelste Interpretation der Beobachtungen. In praktischer Hinsicht ist davon auszugehen, dass zwischen o und etwa 40 fr. $\mathrm{H}^{\circ}$ die $\mathrm{Ca}-\mathrm{Härte}$ bei der $\mathrm{Ab}$ schätzung der Kontaktzeit ungefähr wie folgt zu berücksichtigen ist: Zu der bei o fr. $\mathrm{H}^{\circ}$ sich ergebenden Kontaktzeit (vgl. Konzentrations-Wirkungs-Kurve in destilliertem $\mathrm{H}_{2} \mathrm{O}$ gemäss $\mathrm{Abb}$. 3 ) ist pro fr. $\mathrm{H}^{\circ}$ ein Zuschlag von rund $30 \mathrm{~min}$ (bei $20^{\circ} \mathrm{C}$ ) zu machen. Beispiel: Kontaktzeit bei einer bestimmten Temperatur und $\mathrm{pH}-\mathrm{Wert}=$ IOO min gemäss KonzentrationsWirkungs-Kurve. Härte des Wassers $=30 \mathrm{fr} . \mathrm{H}^{\circ}$. Zuschlag somit $3 \times 30$ $=90 \mathrm{~min}$. Kontaktzeitalso $100+90=190 \mathrm{~min}$. Diese Approximation dürfte für praktische Zwecke genügend genau sein, auch wenn wir mangels spezieller Experimente noch nicht wissen, ob der Ca-Einfluss im ganzen Temperatur- und $\mathrm{pH}$-Bereich immer gleich gross ausfällt.

OBERZILL [II] beschreibt eine Experimentreihe, in welcher Abtötungsversuche mit $E$. coli in verschieden stark mineralisierten Wässern nach gleichem Silberzusatz vorgenommen wurden. Er fand mit zunehmendem Mineralgehalt des Wassers beträchtliche Zunahmen der Kontaktzeiten. Der Effekt ist allerdings schwer zu interpretieren, da unter anderem sowohl $\mathrm{Ca}^{+2}, \mathrm{Mg}^{+2}$ und $\mathrm{Cl}^{-}-$Konzentrationen in den einzelnen Lösungen simultan änderten. Bei zwei Versuchen mit einem $\mathrm{Ca}^{+2}$-Gehalt von $58 \mathrm{bzw} .86 \mathrm{mg} / \mathrm{l}$ und Chloridgehalten von $2 \mathrm{bzw} .4 \mathrm{mg} / \mathrm{l}$ resultierte eine deutlich grössere Kontaktzeit beim höheren $\mathrm{Ca}^{+2}$-Gehalt. Qualitativ stimmt unsere Beobachtung also mit den Resultaten von OBERZILI überein.

Soweit aus den wenigen Angaben von SüPfle und WERNER [Is] in quantitativer Hinsicht abgeschätzt werden kann, fanden sie eine stärkere Hemmung der Bakterizidie durch Härteionen als wir. Ihre Experimente 
Tabelle 9

$V$ ergleich der Abtötungsgescbwindigkeiten in sauerstoffhaltiger und sauerstofffreier Ag-Lösung für E. coli nacb aerober Vorkultur

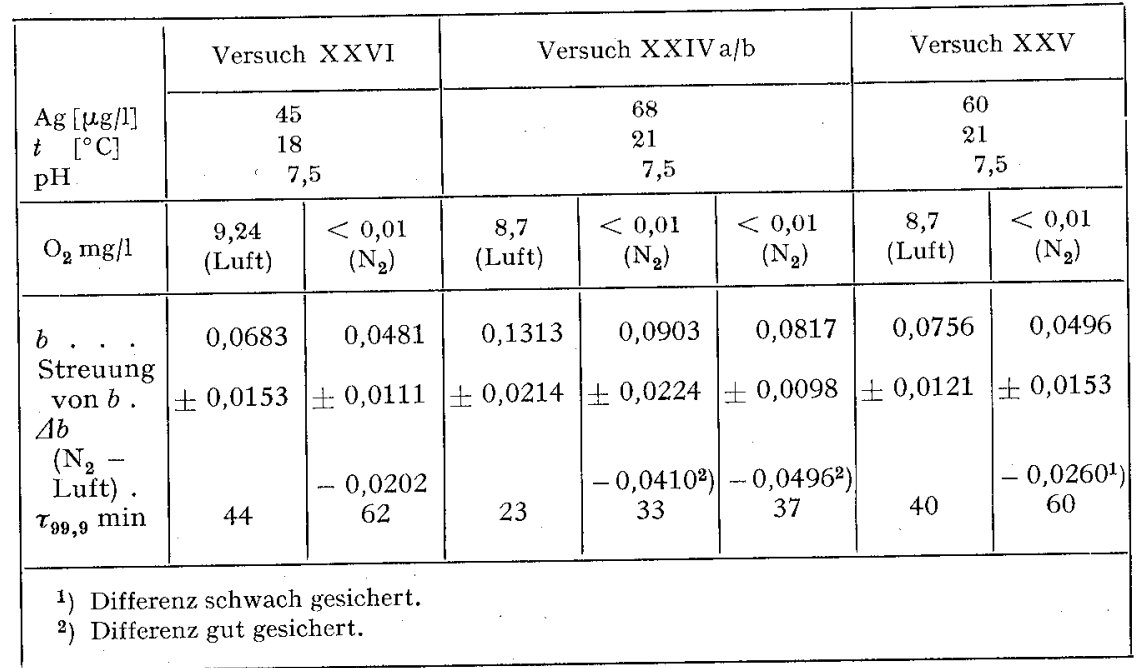

Tabelle 10

Vergleich der Abtötungrgescbwindigkeiten in sauerstoffbaltiger und sauerstofffreier Ag-Lösung für E. coli nach anderober Vorkultur

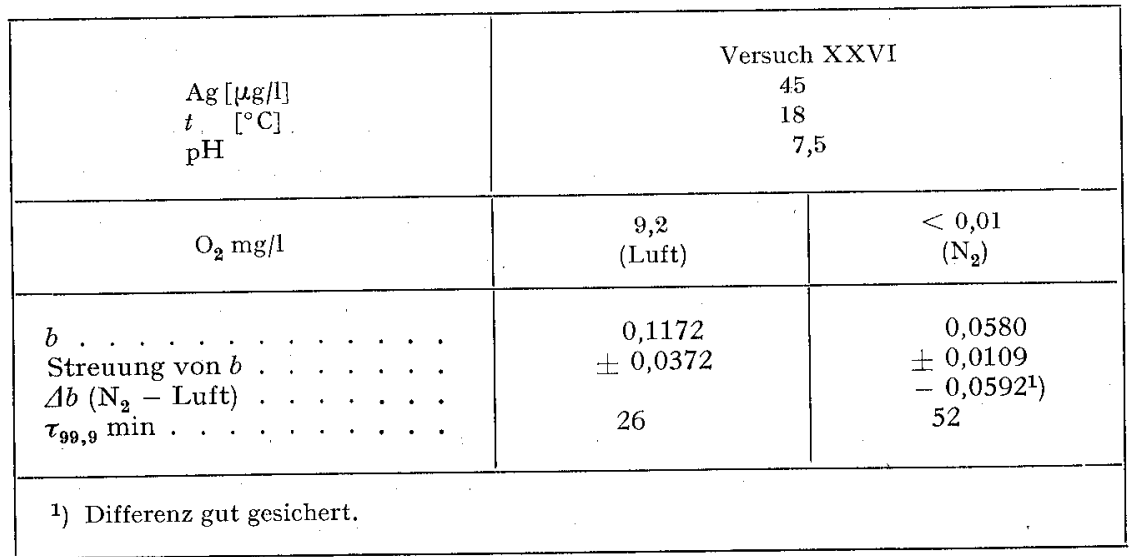


lassen aber aus verschiedenen Gründen (zum Beispiel keine pH-Messung) keinen direkten Vergleich mit unseren Versuchen zu, so dass wir auf eine weitere Diskussion dieses Problems zunächst verzichten müssen.

\subsection{Einfluss des Sauerstoffgehaltes}

Alle Versuche zu diesem Thema wurden wiederum mit Lösungen vorgenommen, die Silber ausschliesslich in ionaler Form enthielten $\left(\mathrm{AgNO}_{3}\right)$. Die Desinfektionslösungen wurden bei den in Tabelle 9 wiedergegebenen Experimenten vor dem Zusatz der Bakteriensuspension mittels Durchleiten von Luft mit Sauerstoff gesättigt bzw. mittels Begasung mit $\mathrm{O}_{2}$-freiem $\mathrm{N}_{2}$ (gereinigt mit Kupferkontakt) vollständig von Sauerstoff befreit. Die Gasdurchleitung wurde während der ganzen Kontaktzeit beibehalten. Für die meisten Versuche dienten coli-Bakterien, die in üblicher Weise unter aeroben Bedingungen vorgezüchtet wurden. Tabelle Io liegt zusätzlich ein Versuch zugrunde, bei welchem der gleiche Bakterienstamm wie beim Versuch von Tabelle 9, aber nach anaerober Vorkultur, zur Prüfung diente.

Aus den Tabellen 9 und ro geht hervor, dass bei dem fakultativ anaeroben $E$. coli unter den bisher geprüften Bedingungen eine etwas geringere Absterbegeschwindigkeit in ganz sauerstofffreien Silberlösungen auftritt als in sauerstoffhaltigen Lösungen. Diese Differenz in der Wirkungsgeschwindigkeit des Silbers scheint bei anaerob vorgezüchteten Bakterien etwas grösser zu sein als bei aerober Vorkultur. Eine Sicherung dieser Beobachtungen durch weitere Experimente scheint uns aber unerlässlich zu sein, um ein eindeutiges Urteil über die Zusammenhänge zwischen $\mathrm{O}_{2}$-Spannung des Milieus und der Abtötungswirkung von Silber zu gewinnen.

Wir möchten vorläufig unsere Beobachtungen nicht als stichhaltigen Beweis dafür betrachten, dass die Silberwirkung in wesentlichem Umfange beeinflusst wird, wenn die Bakterien vom aeroben zum anaeroben Stoffwechsel übergehen. Nun haben allerdings zum Beispiel HerzBerg [8] und GRUMBACH [6] Versuche veröffentlicht, aus denen der Schluss gezogen wurde, dass die Schwermetallwirkung auf Bakterien nur unter aeroben Bedingungen zustande komme. Diese Behauptung wurde auch von ZIMMERMANN [19], allerdings ohne experimentelle Belege, aufgestellt. Bei genauerer Prüfung der von Herzberg und Grumbach verwendeten experimentellen Methoden kommt man aber zum Schlusse, dass neben der Sauerstoffspannung im Milieu eine ganze Reihe von anderen Faktoren die Wirkung des Silbers auf die Bakterien stark beeinflussen musste. Nach unserer Auffassung lässt sich deshalb die von den Autoren gezogene Schlussfolgerung mit ihren Versuchen nicht stützen. 


\section{Empfindlichkeit verschiedener Bakterienarten gegenüber Silber}

Wir haben uns mit dieser Frage nicht sehr eingehend beschäftigt. Aus der Arbeit von HERRMANN [7] lassen sich annähernd quantitative Angaben für eine Reihe von Arten ableiten, wobei allerdings ein strenger Vergleich

\section{Tabelle 11}

Vergleich der Abtötungsgescbmindigkeiten perscbiedener Keimarten. Abtötungszeiten in Minuten für 99,9\% der Keime

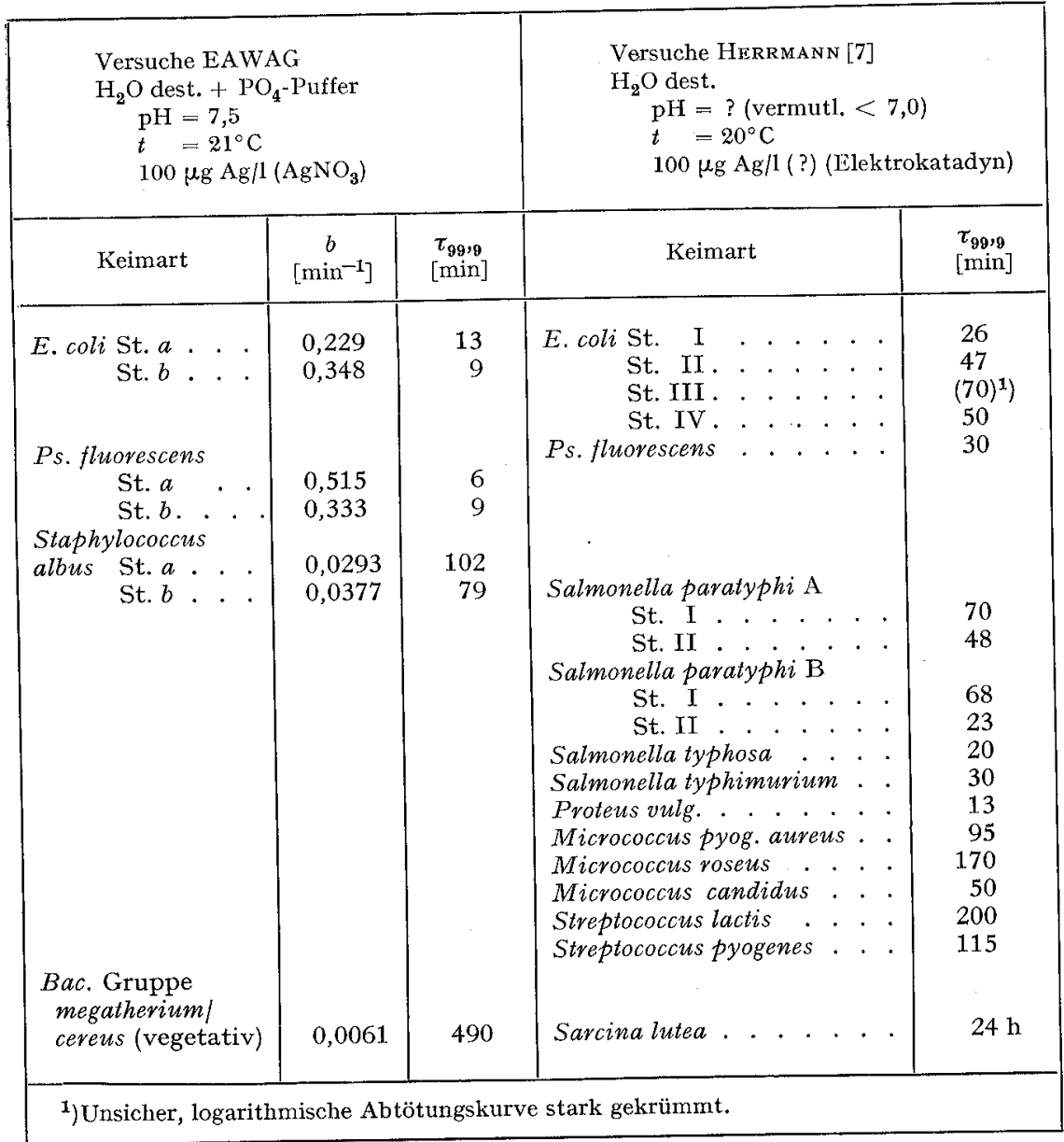


mit unseren Beobachtungen nicht möglich ist, da die Versuchsbedingungen von Her RMANN nicht eindeutig definiert wurden (zum Beispiel keine $\mathrm{pH}$ Angabe) und er auch nur wenige Punkte einer Absterbekurve beobachtete. Weitere quantitative Angaben gibt ferner OBERZILL [II] für 3 colis Stämme, I Typhus- und I Paratyphus-Stamm und 3 nicht näher spezifizierte Stämme « ubiquitärer Wasserkeime».

Qualitative Beurteilungen von Empfindlichkeitsunterschieden verschiedener Bakterienarten finden sich in Arbeiten von Peragallo [I2], TraCY [I6], GoETz et al. [s] und ALHA [I]. In der ausführlichen Arbeit von AlHa ist besonders das Verhalten einer grösseren Zahl pathogener Arten qualitativ beschrieben.

Ergebnisse unserer mehr orientierenden Kontrollversuche mit einigen Saprophythen finden sich in Tabelle Ir. Gleichzeitig haben wir zum Vergleich die von uns aus den Tabellen von HerrmanN berechneten Abtötungszeiten für weitere Arten in Tabelle I2 aufgenommen. Die Zusammenstellung führt zu folgenden Schlüssen:

a) Man muss damit rechnen, dass überaus grosse Empfindlichkeitsunterschiede zwischen verschiedenen Arten vegetativer Keime vorhanden sind. Vergleiche auf vorwiegend qualitativer Basis, wie sie von vielen Autoren gemacht werden, können unter Umständen sehr irreführend sein.

b) Die Verursacher typischer «Wasserkrankheiten» (zum Beispiel Salmonellengruppe) sind mindestens so silberempfindlich wie E. coli (Vergleich innerhalb der Versuche von HERRMANN und OBERZILL); leider fehlen zurzeit Angaben über $V$. cholerae und $S h$. dysentheriae.

c) Es besteht ein auffallender Unterschied zwischen unseren Angaben und denjenigen von HERRMANN über die Abtötungszeiten bei E.coli und Ps. Anorescens. Die Differenzen sind wohl in erster Linie auf Unterschiede im $\mathrm{pH}$-Wert der geprüften Silberlösungen zurückzuführen.

Im Hinblick auf die Forderungen der Wasserversorgungstechnik wäre es erwünscht, wenn unter streng vergleichbaren Versuchsbedingungen, die sich den Verhältnissen der Praxis annähern (speziell bezüglich pH, Mineralisation und Temperatur des Wassers), die Erreger der wichtigsten, von Wasser häufig übertragenen Krankheiten, nochmals quantitativ verglichen werden könnten. Es scheint uns notwendig zu sein, solche Experimente mit verschiedenen Stämmen mindestens folgender Organismen durchzuführen: S. typhosa, S. paratypbi (verschiedene Gruppen), V. colerae, Sh. dysentberiae, Streptococcen (verschiedene Gruppen) und zum Vergleich E. coli. 


\section{Kontrollversuche in Anlehnung an praktische Verhältnisse}

Im Anschluss an die vorstehend beschriebenen Laboratoriumsversuche, die in erster Linie zur Abklärung einiger grundlegender Fragen dienten, haben wir eine Reihe von Experimenten durchgeführt, die ganz auf die praktischen Bedingungen bei der Desinfektion von Wasser mit Hilfe eines der auf dem Markt angebotenen Verfahren abgestimmt wurden. Es war das Ziel dieser Versuche, festzustellen, ob unter praktischen Verhältnissen die in vitro feststellbaren Konzentrations-Wirkungs-Beziehungen ebenfalls beobachtet werden können, und einen Vergleich dieser Beziehungen mit den in Abschnitt 2 und 3 gefundenen Resultaten vorzunehmen.

Als Beispiel wählten wir die elektrolytische Silberung von Wasser mit dem Elektrokatadymerfabren, da es genaue Dosierungen des Silbers besonders leicht macht. Wir beschränken uns darauf, nur eine einzige grössere Ver-

Tabelle 12

Absterbegeschoindigkeit und Kontaktzeiten bei Versuch XXXIV/XXXV mit Elektrokatadynapparat

$t==12^{\circ} \mathrm{C}, \mathrm{pH}=7,5$, Leitungswasser, Ges.-Härte $=20$ frz. $\mathrm{H}^{\circ}, E$. coli, Stamm III, Anfangskeimzahl $=$ rund $10000 / \mathrm{cm}^{3}$.

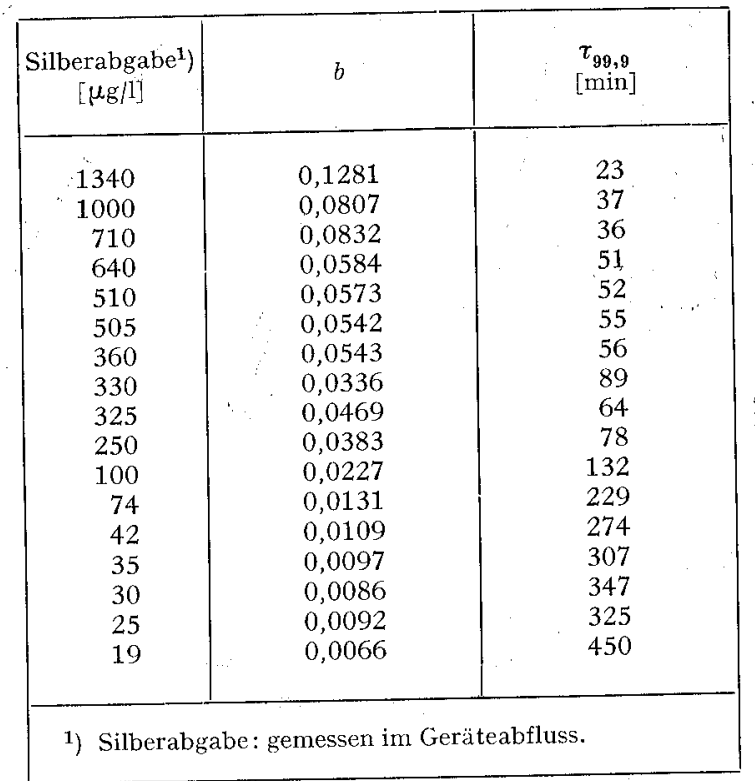




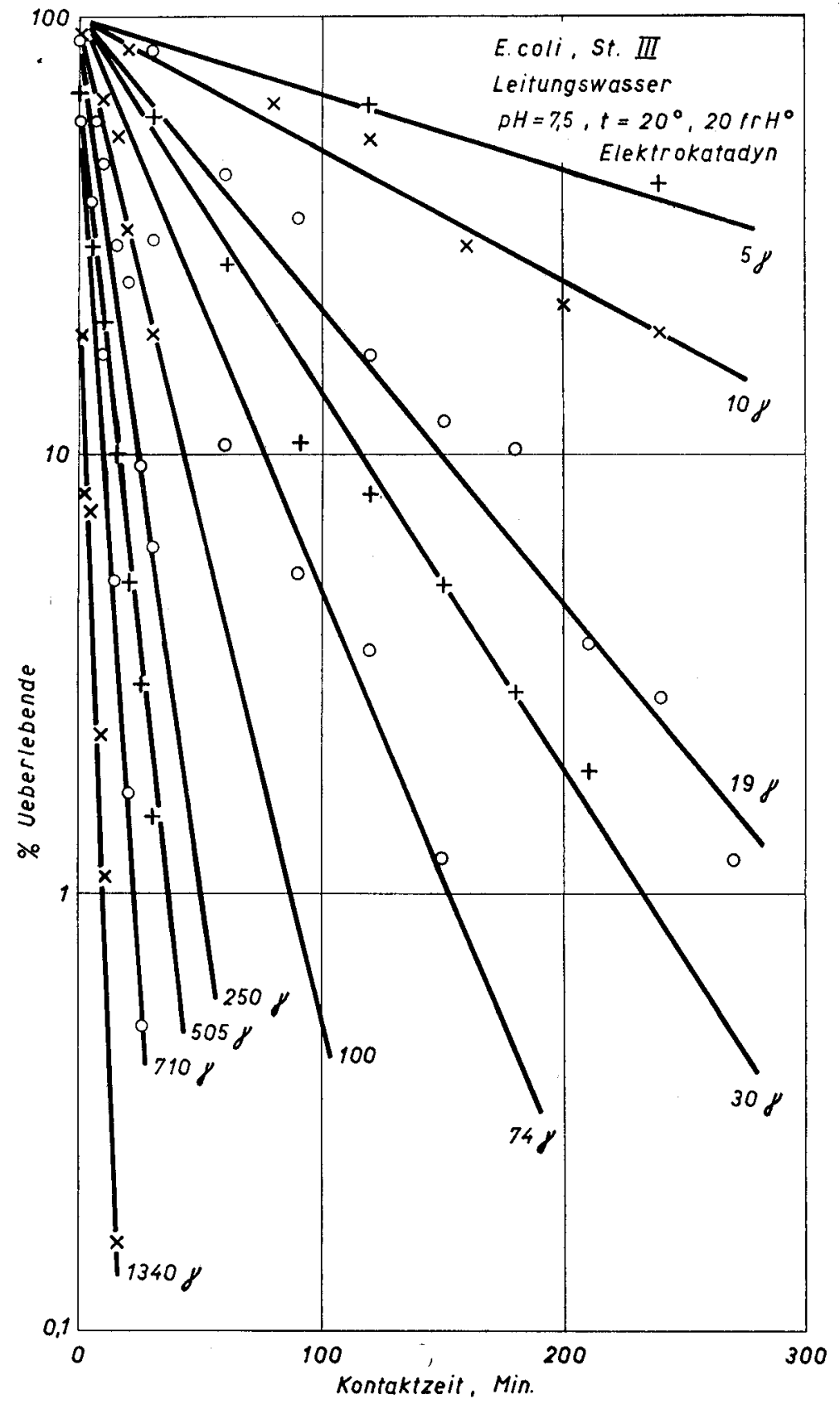

Abbildung 7

Absterbekurven von $E$. coli im Ablauf eines Elektrokatadyngerätes in Funktion der Zeitdauer zwischen Durchlauf des Wassers an den Elektroden und Probenahme. Jede Kurve entspricht einer bestimmten "Aktivierung» (Silberkonzentration). 
suchsserie darzustellen, da die verschiedenen Experimente nichts grundsätzlich Neues brachten.

Metbode: Ein etwa $\mathrm{I} \mathrm{m}^{3}$ haltender Tank wurde mit Leitungswasser gefuillt und mit einigen Kubikzentimetern einer coli-Kultur in MilchzuckerNährlösung beimpft. Der Tankinhalt wurde dann mittels einer Pumpe durch einen Elektrokatadyn-Apparat (Typ 24) gefördert und der Abfluss des Gerätes in sterilen Behältern aufgefangen. Die Silberdosierung erfolgte mittels Variation der Stromstärke und des Wasserdurchflusses. Das Wasser wurde unmittelbar nach Verlassen des Gerätes sowie nach verschiedenen Kontaktzeiten bakteriologisch untersucht. Das nachstehend beschriebene Experiment wurde mit einem nicht näher identifizierten Stamm aus der coli-Gruppe (Stamm III) vorgenommen, der aus Wasser isoliert war.

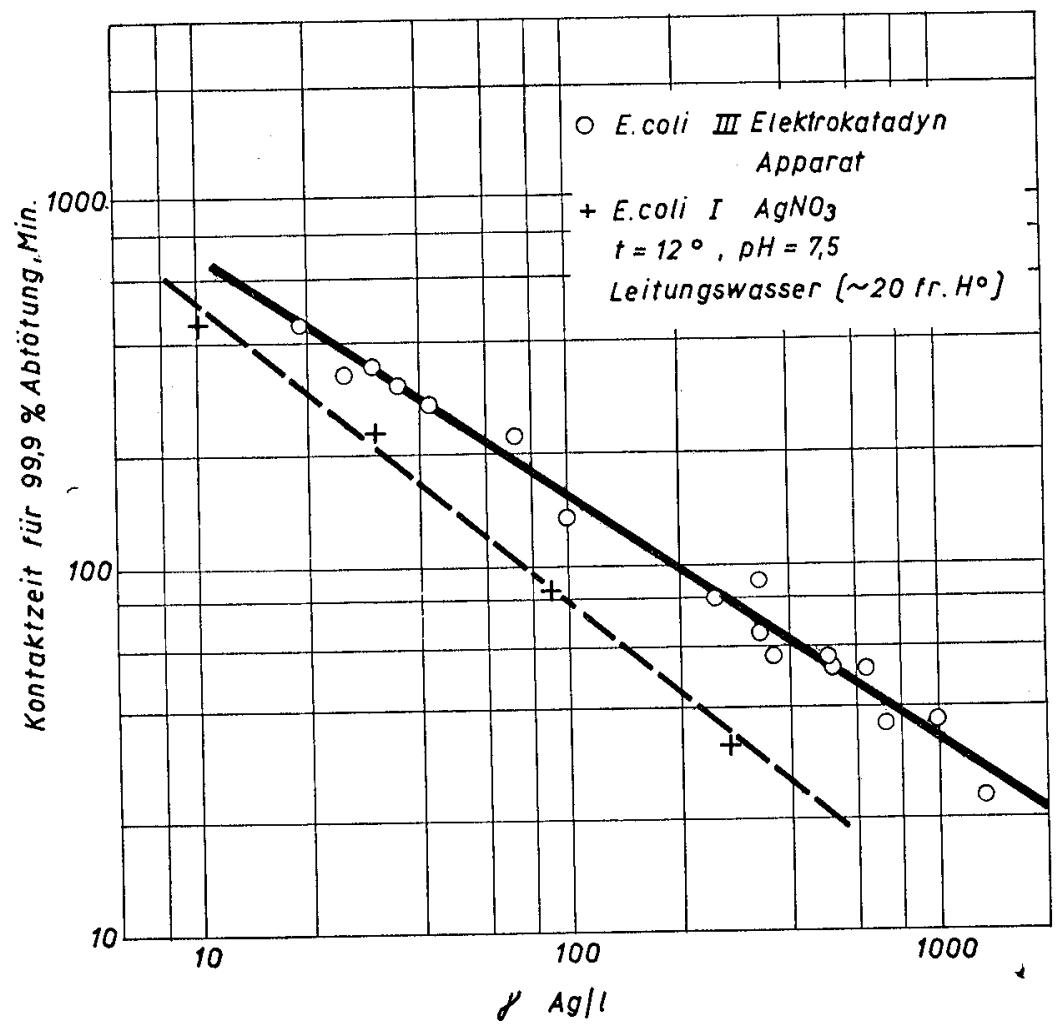

Abbildung 8

Konzentrations-Wirkungs-Kurve von Silberlösungen aus einem Elektrokatadyn-Gerät für E.coli III(O). ZumVergleich die im Faktorenversuch (Abb. 3) gefundene Kurve für E. coli I, korrigiert für die Bedingungen des Katadynversuches $(+)$. 
Ergebnisse: Wie sich aus Abbildung 7 ergibt, erfolgt das Absterben der Bakterien im silberhaltigen Abfluss des Katadyn-Apparates in durchaus analoger Weise wie in den Laboratoriumsversuchen mit Silbernitrat. Die Keimverminderung, die innerhalb des Gerätes selbst eintritt, ist erwartungsgemäss eine Funktion der Durchflusszeit und der Silberkonzentration. Eine Keimabnahme, die praktisch ins Gewicht fällt, tritt beim Gerätedurchfluss nicht ein, es sei denn, es werde eine sehr hohe Silberabgabe eingestellt. Im Laufe der anschliessenden Kontaktzeit tritt eine Keimverminderung gemäss einer Reaktion erster Ordnung ein, wie sie auch in den $\mathrm{AgNO}_{3}$-Lösungen beobachtet wurde. In Tabelle I2 sind die Geschwindigkeitskonstanten der Absterbekurven zusammen mit den daraus errechneten Kontaktzeiten für 99,9\% Keimabtötung bei verschiedenen Silberdosierungen zusammengefasst.

Die Konzentrations-Wirkungs-Kurve, die sich aus den vorstehenden Messwerten ergibt, ist in Abbildung 8 aufgezeichnet. Man erhält wiederum eine streng lineare Beziehung zwischen logarithmischer Konzentration und logarithmischer Kontaktzeit, wie in den früheren Experimenten. Wir haben in die Abbildung 8 auch die Konzentrations-Wirkungs-Kurve aus dem Faktorenversuch (Abschnitt 3) bei pH 7,5 eingetragen, wobei die notwendigen Korrekturen für die Temperatur von $12^{\circ}$ und die Wasserhärte von $20 \mathrm{frz} . \mathrm{H}^{\circ}$ rechnerisch angebracht wurden. Es zeigt sich, dass der beim Faktorenversuch verwendete coli-Stamm wesentlich empfindlicher war als derjenige des Temperaturversuches (vor allem in den hohen Ag-Konzentrationen). Dabei muss allerdings noch berücksichtigt werden, dass beim Tankversuch das verwendete Leitungswasser durch die Nährlösung der zugesetzten Impfsuspension etwas verunreinigt wurde und damit, ähnlich wie unter praktischen Verhältnissen, eine gewisse Verminderung der Silberwirkung eintreten konnte.

Wir schliessen aus dem vorstehenden Vergleich, dass die Gesetzmässigkeiten der Silbermirkung, die in den Laboratoriumspersucben gefunden wurden, ibre Gültigkeit ermartungsgemäss auch unter praktischen Bedingungen beibebalten. Wie wir bereits festgestellt haben, wären aber genauere Angaben über die relative Empfindlichkeit der häufigsten Keimarten für die Praxis zweifellos erwünscht und in späteren Versuchen noch zu ermitteln.

\section{Zusammenfassung}

a) Die bakterizide Wirkung von Silber in wässrigen Lösungen wurde in Abhängigkeit der Silberkonzentration, der Temperatur, des pH-Wertes 
und der Konzentration von $\mathrm{Cl}^{-}, \mathrm{PO}_{4}^{-3}, \mathrm{Ca}^{+2}$ und Sauerstoff geprüft. Testorganismus war E. coli. Die Experimente erfolgten unter peinlichst genau festgelegten Bedingungen, und nur statistisch auswertbare und miteinander vergleichbare Versuchsreihen wurden berücksichtigt. Die Resultate führen $\mathrm{zu}$ folgenden Schlüssen:

Die Absterbeordnung von E. coli (und anderer Arten) in Ag-Lösungen ist von I. Ordnung.

Die Beziehung zwischen $\log$ (Ag-Konzentration) und $\log$ (Kontaktzeit) für eine bestimmte Abtötungsrate ist linear.

Für den Temperatur- und $\mathrm{pH}$-Einfluss auf die Abtötungsgeschwindigkeit von $E$. coli wurden konstante Werte gefunden, die voneinander und von der Silberkonzentration unabhängig sind. Sie betragen für die Temperatur $\Theta_{10}=\mathrm{I}, 6$ und für den $\mathrm{pH}$-Wert $\Pi_{1}=\mathrm{I}, 55 . \mathrm{Cl}^{-}-\mathrm{PO}_{4}^{-3}$ - und $\mathrm{Ca}^{+2}$-Ionen hemmen die bakterizide Wirkung von Silber. Es wird festgestellt, dass die Reaktionsprodukte zwischen $\mathrm{Ag}^{+}$und $\mathrm{Cl}^{-}$zum Teil noch bakterizid wirken. Eine Abhängigkeit der bakteriziden Wirkung von Silber von der Sauerstoffspannung in den Lösungen konnte nicht nachgewiesen werden.

Die Bakterizidie von $\mathrm{AgNO}_{3}$-Lösungen ist identisch mit derjenigen gleich konzentrierter, elektrnlytisch aus Silbermetall hergestellter Lösungen.

b) Es wird das Problem der Empfindlichkeitsunterschiede verschiedener Keimarten in Silberlösungen anhand eigener Versuche und von Literaturangaben diskutiert.

c) Im Hinblick auf die Praxis der Wasserdesinfektion wird im Sinne eines Beispiels eine Versuchsreihe mit einem sogenannten Elektro-Katadynapparat dargestellt. Es zeigt sich, dass die Entkeimung von Wasser, das ein solches Gerät durchflossen hat, nach den genau gleichen Gesetzmässigkeiten erfolgt, wie sie in Laboratoriumsexperimenten mit anderen Silberlösungen gefunden wurden.

\section{LITERATURVERZEICHNIS}

[1] Alha, A. R., Acta Path. Microbiol. Scand. Suppl. Nr. 65 (1947).

[2] BROWNLEE, K. A., Industrial Experimentation (Her Majest. Stat. Off., London I949).

[3] Fischer, H., und Leopoldi, Z. Anal. Chem. 101, I (1935).

[4] GAUze, G. F., C. R. Acad. Sci. USSR 27, 722 (1940); vgl. Brit. Abstr. [A] 3, 230 (1946). 
[s] Goetz, A., Tracy, R. L., und Harris, F. S., in: Silper in Industry. Herausgegeben von L. AdDicks (Reinhold Publ. Corp., New York 1940).

[6] Grumbach, A., Schweiz. Z. Path. Bakt. 9, 395 (1946); 11, 504 (1948); und 12, 97 (1949).

[7] Herrmann, A., Diss. (ETH, Zürich 1934).

[8] HerzberG, K., Zbl. Bakt. [I] 90, II 3 (I923).

[9] LINDER, A., Statistiscbe Metboden für Naturwissenscbafter, Mediziner und Ingenieure (Verlag Birkhäuser, Basel 195r).

[Io] Oberzill, W., Sci. Pharm. 24, 17I (1956).

[i I] Oberzill, W., Arch. Hyg. Bakt. 141, 2I7 (I957).

[i2] Peragallo, J., Intern. Bericht, Katadyn-Gesellschaft (1936).

[13] RaO, C. R., Adpanced Statistical Metbods in Biometric Research (Wiley, New York I952), S. 86.

[14] Romans, J. B., in: Antiseptics, Desinfectants, Fungicides and Desinfection (Lea and Febiger, Philadelphia I954).

[I s] SÜPFLE, K., und Werner, R., Mikrochem. 36/37, 866 (I950).

[I6] Tracy, R. L., J. Bact. 41, 33 (I94I).

[17] WuhrmanN, K., und Meyrath, J., Schweiz. Z. Path. Bakt. 18, 1060 (1955).

[I8] WuhrmanN, K., Schweiz. Z. Hydrol. 29, I08 (1957).

[I9] ZimmermanN, W., Z. Hyg. 135, 403 (1952).

[20] Снick, H., J. Hyg. 8, 92 (I908). 